\title{
Testing the individual and social learning abilities of task- naïve captive chimpanzees (Pan troglodytes sp.) in a nut- cracking task
}

\author{
Damien Neadle ${ }^{\text {Corresp., 1, } 2}$, Elisa Bandini ${ }^{3}$, Claudio Tennie ${ }^{3}$ \\ ${ }^{1}$ School of Psychology, College of Life and Environmental Sciences, University of Birmingham, Birmingham, United Kingdom \\ 2 Department of Psychology, School of Social Sciences, Faculty of Business, Law and Social Sciences, Birmingham City University, Birmingham, United \\ Kingdom \\ 3 Department of Early Prehistory and Quaternary Ecology, Eberhard-Karls-Universität Tübingen, Tübingen, Germany \\ Corresponding Author: Damien Neadle \\ Email address: damienneadle@outlook.com
}

Nut-cracking is often cited as one of the most complex behaviours observed in wild chimpanzees. However, the cognitive mechanisms behind its acquisition are still debated. The current null hypothesis is that the form of nut-cracking behaviour relies on variants of social learning, with some researchers arguing, more precisely, that copying variants of social learning mechanisms are necessary. However, to date, very few experiments have directly investigated the potentially sufficient role of individual learning in explaining the behavioural form of nut-cracking. Despite this, the available data provides some evidence for the spontaneous acquisition of nut-cracking by chimpanzees; later group acquisition was then found to be at least facilitated by (unspecified) variants of social learning. The latter findings are in line with both suggested hypotheses, i.e., that copying social learning is required and that other (non-copying) social learning mechanisms are at play. Here we present the first study which focused (initially) on the role of individual learning for the acquisition of the nut-cracking behavioural form in chimpanzees. We tested task-naïve chimpanzees $(\mathrm{N}=13)$ with an extended baseline condition to examine whether the behaviour would emerge spontaneously. After the baseline condition (which was unsuccessful), we tested for the role of social learning by providing social information in a step-wise fashion, culminating in a full action demonstration of nut-cracking by a human demonstrator (this last condition made it possible for the observers to copy all actions underlying the behaviour). Despite the opportunities to individually and/or socially learn nut-cracking, none of the chimpanzees tested here cracked nuts using tools in any of the conditions in our study; thus, providing no conclusive evidence for either competing hypothesis. We conclude that this failure was the product of an interplay of factors, including behavioural conservatism and the existence of a potential sensitive learning 
period for nut-cracking in chimpanzees. The possibility remains that nut-cracking is a behaviour that chimpanzees can individually learn. However, this behaviour might only be acquired when chimpanzees are still inside their sensitive learning period, and when ecological and developmental conditions allow for it. The possibility remains that nutcracking is an example of a culture dependent trait in non-human great apes. Recommendations for future research projects to address this question are considered. 


\section{Testing the individual and social learning abilities of}

\section{2 task-naïve captive chimpanzees (Pan troglodytes sp.)}

3 in a nut-cracking task

4

5

6
Damien Neadle ${ }^{1,2}$, Elisa Bandini ${ }^{3}$ and Claudio Tennie ${ }^{3}$

${ }^{1}$ School of Psychology, College of Life and Environmental Sciences, University of Birmingham, Birmingham, United Kingdom

${ }^{2}$ Department of Psychology, School of Social Sciences, Faculty of Business, Law and Social Sciences, Birmingham City University, Birmingham, United Kingdom

${ }^{3}$ Department of Early Prehistory and Quaternary Ecology, Eberhard-Karls-Universität Tübingen, Tübingen, Germany

\section{Corresponding Author:}

Damien Neadle ${ }^{1,2}$

Psychology Department

Birmingham City University

The Curzon Building

4 Cardigan Street

Birmingham

B4 7BD

United Kingdom

Email address: Damien.Neadle@bcu.ac.uk 


\section{Abstract}

Nut-cracking is often cited as one of the most complex behaviours observed in wild

31 chimpanzees. However, the cognitive mechanisms behind its acquisition are still debated. The current null hypothesis is that the form of nut-cracking behaviour relies on variants of social learning, with some researchers arguing, more precisely, that copying variants of social learning mechanisms are necessary. However, to date, very few experiments have directly investigated the potentially sufficient role of individual learning in explaining the behavioural form of nutcracking. Despite this, the available data provides some evidence for the spontaneous acquisition of nut-cracking by chimpanzees; later group acquisition was then found to be at least facilitated by (unspecified) variants of social learning. The latter findings are in line with both suggested hypotheses, i.e., that copying social learning is required and that other (non-copying) social learning mechanisms are at play. Here we present the first study which focused (initially) on the role of individual learning for the acquisition of the nut-cracking behavioural form in chimpanzees. We tested task-naïve chimpanzees $(\mathrm{N}=13)$ with an extended baseline condition to examine whether the behaviour would emerge spontaneously. After the baseline condition (which was unsuccessful), we tested for the role of social learning by providing social information in a step-wise fashion, culminating in a full action demonstration of nut-cracking by a human demonstrator (this last condition made it possible for the observers to copy all actions underlying the behaviour). Despite the opportunities to individually and/or socially learn nutcracking, none of the chimpanzees tested here cracked nuts using tools in any of the conditions in our study; thus, providing no conclusive evidence for either competing hypothesis. We

50 conclude that this failure was the product of an interplay of factors, including behavioural conservatism and the existence of a potential sensitive learning period for nut-cracking in 
53 individually learn. However, this behaviour might only be acquired when chimpanzees are still

54 inside their sensitive learning period, and when ecological and developmental conditions allow

55 for it. The possibility remains that nut-cracking is an example of a culture dependent trait in

56 non-human great apes. Recommendations for future research projects to address this question are

57 considered. 


\section{Introduction}

Humans have created a unique niche within the animal kingdom, one that (most likely)

61 relies on an ability to transfer knowledge between and within generations, allowing our species

62 to inhabit almost every environment on the planet. However, modern industrialised human

63 society is so far removed from that of our ancestors, that it is difficult to understand how our

64 cultural ability - or our cultural niche (Odling-Smee, Laland, \& Feldman, 2003) - evolved.

65 Therefore, the closest extant relatives of the hominin clade, non-human great apes (hereafter:

66 apes), are often used as a phylogenetic proxy to help shed light on our own evolution. Modern human culture may be unique in the animal kingdom (Tomasello, 1998) - at least in the technological domain (Tennie, Caldwell, \& Dean, 2018). Thus, to allow for the study of culture across species, a more minimal (or soft) definition of culture is required. Here we follow the terminology suggested recently by Neadle, Allritz and Tennie (2017), in which a cultural trait is any behaviour that is at least influenced (including merely its frequency being facilitated or catalysed; Tennie, Call, \& Tomasello, 2010) by social learning. Within this definition of culture, the social learning mechanisms at play can be any of the many proposed variants (for an overview, see Whiten, Horner, Litchfield, \& Marshall-Pescini, 2004). This minimal definition of culture allows for the identification of cultures that involve a range of social learning variants, including ones that rely on the copying of behaviour directly (often called imitation, see, e.g., Whiten \& Ham, 1992). Crucially, the minimal definition of culture also encompasses examples of behavioural forms that can emerge without requiring social learning. Instead, behaviours that rely on (i.e., cannot occur in the absence of) copying variants

80 of social learning are 'culture dependent traits' (henceforth CDTs; see Reindl, Apperly, Beck, \&

81 Tennie, 2017; Tennie, Caldwell, \& Dean, 2018). Some animal behaviours and artefacts may be 82 culture dependent (e.g., whale song is a candidate CDT; Tennie et al., 2018), but whether any 
83 animal tool use qualifies as CDT is a matter of considerable debate (Galef, 1992; Kendal, 2008;

84 Tennie, Call, \& Tomasello, 2009). In the human case, technology has often evolved to the point

85 that no naïve individual could reinnovate the behaviour on their own within their lifetime (Galef,

86 1992; Tomasello, Kruger, \& Ratner, 1993), making many modern human traits CDTs and indeed

87 examples of cumulative culture (Boyd \& Richerson, 1996). For a more in-depth discussion of

88 these, and other, terms we refer the reader to a glossary of terms in the supplementary materials

89 provided.

Amongst non-human animals (hereafter: animals), chimpanzees (Pan troglodytes) are,

91 for now, the 'most cultural' species - at least in terms of (known) numbers of cultural traits

92 (Whiten et al., 1999) - where a mere increase in sheer number of cultural traits is known as

93 accumulation (Dean, Vale, Laland, Flynn, \& Kendal, 2014). However, accumulation (numbers of

94 traits) should not be confused with cumulation, i.e. the cultural change of the traits themselves

95 along transmission pertaining to cumulative culture (Dean et al., 2014). Only the latter is the

96 result of the 'ratchet effect' (Tomasello et al., 1993), which underlies cumulative culture (Boyd

$97 \&$ Richerson, 1996) and is responsible for CDTs (Reindl et al., 2017), which are the product of

98 cumulative culture. Whilst chimpanzees' number of cultural traits is impressive, for those

99 concerned with human cultural evolution, the presence or absence of CDTs in chimpanzees is of

100 particular interest.

101 Regarding chimpanzee culture, nut-cracking is considered one of the most complex

102 behaviours expressed by any wild apes. Complexity can refer to the number of parts within a

103 final artefact/behaviour (techno-units; Oswalt, 1976), the goals and sub goals of an action (Read

$104 \&$ Andersson, 2019), the manual dexterity of an action (Foucart et al., 2005) and the number of

105 "rules" necessary to describe the behaviour (Sirianni, Mundry, \& Boesch, 2015), amongst other 
106 metrics (see Vaesen \& Houkes, 2017 for further discussion of complexity). Nut-cracking

107 requires a high level of dexterity (Foucart et al., 2005) and involves several tools in various steps

108 that need to be followed in a specific sequential order to produce the desired effect (Biro et al.,

109 2003; Boesch, Bombjaková, Meier, \& Mundry, 2019; Inoue-Nakamura \& Matsuzawa, 1997;

110 Read \& Andersson, 2019); thus, it can be considered a complex behavioural form. Furthermore,

111 nut-cracking is rare across wild communities, (so far) only being documented in two

112 geographically separate populations: two communities in West Africa (Bossou, Guinea and Taï

113 Forest, Côte d'Ivoire Whiten et al., 2001) and one in Ebo Forest, Cameroon (Morgan \& Abwe,

114 2006; although note that these data are based on indirect evidence and should be treated with

115 some degree of caution).

116 The number of steps alongside the manual dexterity and use of multiple objects required

117 for this behaviour suggests that nut-cracking is most likely a complex behaviour for

118 chimpanzees. The basic behavioural form of nut-cracking consists of the following four

119 sequential steps, though note that other steps might occur:

120 1. Place nut on anvil

121 2. Pick up hammer (unless already picked up)

3. Lift hammer up

123 4. Drop/push the hammer onto nut (all may be repeated).

124 Perhaps due to this apparent complexity, nut-cracking is often assumed to be culturally

125 transmitted (Boesch \& Boesch-Achermann, 2000; Lycett, Collard, \& McGrew, 2007, 2010),

126 with some researchers arguing that action copying (or imitation) must be the mechanism

127 responsible for its acquisition. For example, Boesch (1996) claims that chimpanzees learn how to

128 crack nuts "by individual and social learning, including imitation" (Boesch, 1996, p. 418, 
129 emphasis added). Biro et al (2003, p. 220) further argue that when nut-cracking "infant

130 chimpanzees are driven not by a motivation for food but to produce a copy of the mother's

131 actions" [emphasis added]. More generally, others agree, claiming that nut-cracking (alongside

132 other chimpanzee traits) is difficult to explain "by social learning processes simpler than

133 imitation" (Whiten et al., 1999, p. 685). More recently, similar claims have been made that

134 chimpanzees rely on mother to infant "teaching" to acquire the skills required to crack nuts at a

135 rate consistent with that of others within their community (Boesch et al., 2019). Some have

136 further argued that young wild chimpanzees engage with this process during a so-called

137 'sensitive learning period' between the ages of approx. 3.5 years and 10 years (Inoue-Nakamura

138 \& Matsuzawa, 1997; Matsuzawa, 1994; Biro et al., 2003).

139 However, other research has suggested that dispersing primates, outside the estimated

140 sensitive learning period, can still engage with and adopt behaviours - perhaps in keeping with

141 their new groups. Most relevant here, Luncz, Mundry and Boesch (2012) describe how dispersed

142 female chimpanzees adapt their hammer choice during nut-cracking to conform to that of their

143 new group. The dispersed females were beyond the age of sexual maturity, and so outside their

144 sensitive learning period. The fact that these individuals can modify their behaviour in this way,

145 suggests that the possibility remains for individuals outside of the sensitive learning period to

146 adopt the full behavioural form. This said, these findings are not evidence of the behavioural

147 form of nut-cracking emerging for the first time, instead are evidence of behaviours being

148 adapted and therefore whilst interesting might not represent a strong argument against the notion

149 of a sensitive learning period.

150 In conclusion, the behaviours underpinning nut-cracking have been argued to require

151 social learning (in particular action copying and/or unspecified variants of teaching). Therefore, 
152 it has been assumed that nut-cracking is outside of naïve chimpanzees' individual learning

153 abilities, which would make nut-cracking a CDT (sensu Reindl et al., 2017). This is a clear claim

154 that can be tested. If nut-cracking requires social learning (if it is indeed a CDT), it should re-

155 appear when a naïve chimpanzee has access to a model nut cracker to observe, thus suggesting

156 that social learning is required for nut-cracking to occur. However, should we fail to identify nut-

157 cracking, even at the end of this study, this should only be considered as an indication that it

158 might not be a CDT rather than concrete evidence for it. Such a result would need replicating in

159 another population before confident assumptions can be made from the data. Indeed, it is

160 possible that environmental, social or individual factors might influence the likelihood of

161 expression (sensu Tennie et al., 2009). However, if nut-cracking does occur in a baseline

162 condition in this naïve population, this would constitute evidence that naïve chimpanzees have

163 the capacity to reinnovate nut-cracking in the absence of social learning (i.e. it is within the

164 species' ZLS).

165 In one formulation, the Zone of Latent Solutions (ZLS) hypothesis (Tennie et al., 2009)

166 posits that all non-human great ape 'cultural' behaviours can be reinnovated (defined by Bandini

$167 \&$ Tennie, 2017) by naïve apes. This specific case has been termed as the 'ZLS-Only' hypothesis

168 (Reindl, Bandini \& Tennie, 2018). In line with this, Hayashi, Mizuno and Matsuzawa (2005)

169 suggested that nut-cracking could potentially be individually reinnovated by chimpanzees. Some

170 field reports support these views; for example, a report of nut-cracking in Cameroon (Morgan \&

171 Abwe, 2006) passes the 'information barrier' of the N'Zo-Sassandra River (McGrew, Ham,

172 White, Tutin, \& Fernandez, 1997). This report can be regarded as the outcome of a natural quasi-

173 latent solution test (sensu Bandini \& Tennie, 2018), as this pattern strongly suggests that that

174 nut-cracking was (re-)innovated in two, culturally separate, wild communities (Tennie et al., 
1752009 , p. 2406). Though, again, these results should be considered in the light of the fact that they

176 are supported by second hand reports, sounds in the forest and finding of tools.

177 If all underlying steps of the nut-cracking behaviour are also found to be reinnovated by a

178 naïve, captive, chimpanzee in a culturally separate "island" of individuals (Tennie, Braun,

179 Premo, \& McPherron, 2016; Tomasello, 1999) then the behaviour would (by definition) cease to

180 be a putative example of an animal CDT. This would support the ZLS hypothesis and would

181 suggest that chimpanzees are capable, in principle, of individually learning the basic behaviour

182 form underpinning nut-cracking; demonstrating that social learning is not required for this to

183 occur. Importantly, it should be noted that social learning is likely to play a role in the process of

184 chimpanzees understanding that nuts are a food source and, in addition, that they can be

185 considered a 'safe' food (Hopper, Schapiro, Lambeth, \& Brosnan, 2011). Although this process

186 is important for the frequency of nut-cracking within and across populations, our study is

187 concerned with the mechanisms underlying tool-use aspect of the behavioural form of nut188 cracking.

189 Therefore, here we test two competing hypotheses: chimpanzee nut-cracking as a culture

190 dependent trait (the "CDT hypothesis") versus chimpanzee nut-cracking as a behaviour that can

191 be individually learned (re-innovated; Bandini \& Tennie, 2017), but whose expression may

192 nevertheless be facilitated by non-copying variants of social learning (the "ZLS hypothesis",

193 compare Tennie et al., 2009; in press). Simply, the ZLS hypothesis posits that nut-cracking

194 should emerge in a 'baseline' condition, i.e., without requiring social learning. Contrastingly, the

195 CDT hypothesis argues that copying variants of social learning are necessary for the emergence

196 of nut-cracking in a naïve sample. 
198 dependent, have been reinnovated by naïve, captive subjects in latent solution tests (Bandini \&

199 Tennie, 2017, 2019; Menzel, Fowler, Tennie, \& Call, 2013; Motes-Rodrigo et al., 2019; Neadle

200 et al., 2017; Tennie et al., 2009; Tennie, Hedwig, Call, \& Tomasello, 2008). These behaviours

201 ('latent solutions'; Tennie et al., 2009), were reinnovated without requiring any observation (or

202 teaching). This does not, however, mean that social learning does not play any role in the

203 innovation likelihood of these behavioural forms. Indeed, several variants of non-copying social

204 learning (the specific mechanism was not directly tested in the studies mentioned above) greatly

205 facilitate the innovation likelihood of the behaviour in both captive and wild chimpanzees

206 (therefore affecting the observed frequencies of behaviours within and across populations;

207 Tennie et al., in press; Bandini \& Tennie, 2017, 2019).

In the current study, we tested both the CDT and the ZLS hypotheses predictions for nutcracking. In 2010, Tennie et al. hypothesised that nut-cracking would be within the chimpanzee ZLS, but that it may simply have a relatively low baseline probability of reinnovation (i.e., it is at

211 the very edge of the chimpanzee ZLS). We were able to test both hypothesis by applying the

212 extended latent solutions testing methodology (first described in Bandini \& Tennie, 2018). This

213 method first starts by testing for the reinnovation of the target behavioural form (here nut-

214 cracking) in completely naïve chimpanzees (we ensured naivety by asking keepers of the

215 animals' previous experiences of the behaviour) - thus testing the ZLS hypothesis. If the

216 behaviour does not appear in this baseline, subjects are then provided with incremental levels of

217 social learning information. The particular methodology followed in this study allows for the

218 examination of the role of individual learning (initial baseline test), then subsequently for end-

219 state emulation, goal emulation and finally action copying (imitation) in the emergence of the 
220 target trait. By testing these competing hypotheses it is possible to determine whether the

221 reliance on specific variants of social learning is restricted to our own species or whether it is

222 also common to other apes. Should the former be supported by the present study, we would

223 consider that comparisons between ape and modern human cultures should be largely suspended,

224 until evidence of their similarity in using copying mechanisms is established. If the latter were

225 true, it would suggest that the capacity for the resulting type of culture (cumulative culture,

226 leading to CDTs) was shared by the last common ancestor between modern apes and humans.

\section{Materials \& Methods}

\section{Subjects}

The subjects were 13 chimpanzees $\left(\mathrm{M}_{\mathrm{age}}=31.08 ; \mathrm{SD}=1 ;\right.$ female $=9$, male $=4$; See

Table 1; Pan troglodytes sp.). All subjects lived within a single group and comprised the entirety

231 of that group, except for one individual (C13), which, due to group transfers within the

232 zoological institution throughout the duration of this study, was introduced into the group before

233 the start of the second condition (therefore $\mathrm{C} 13$ did not participate in the baseline condition).

234 Subjects were provided with scatter feeds, consisting primarily of vegetables with some fruit in

235 the morning (approx. 10am) and again in the afternoon (approx. 3pm). The subjects were housed

236 in two enclosures throughout the course of the study; between June 2017 and April 2018 subjects

237 were housed in the "conversion" enclosure, from April 2018 until the end of the study subjects

238 were housed in the "Eden" enclosure. Both enclosures consisted of two indoor areas and an

239 outdoor area (two smaller areas in the case of conversion), with separate management areas

240 (away from the observation of visitors). Subjects could be observed through glass panes in all

241 public areas and mesh in management areas, observations used in this study were obtained from

242 both. Within the main enclosures, subjects had access to enrichment devices, such as climbing 
243 frames/ropes, hanging feeders and nesting baskets. Other enrichment devices are regularly

244 provided by keepers.

245

TABLE 1 ABOUT HERE

\section{Prior experience questionnaire}

In order to exclude any possible influence of social learning on the results of this study,

keepers filled out questionnaires and were interviewed (designed and distributed by EB at the

zoological institution) about prior tool use behaviour. The use of this questionnaire was approved

by the University of Birmingham STEM ethical review committee (ERN_17-1729). The

questionnaire asks keepers to provide details on behaviours relating to "Using one object to bang

on, or hit, another: usually, this means the use of a hard object to bang on or hit another, often

hard, object. This may be with the aim to crack or break open the latter object, or to remove a

256

257

substrate. Here, we are interested in any hammer-like behaviours, regardless of the objects

involved". This definition encompasses nut-cracking and similar actions, such as hammering

behaviours. No instances of nut-cracking were reported in the questionnaire; however, a keeper

described how one individual (C6; female; age 9) used a stone to tap on the glass of the outdoor enclosure. All but one keeper reported that the chimpanzees were frequently witnessed using their teeth to crack nuts, although they have never been provided with shelled macadamia nuts.

\section{Ethical statement}

All participation in this study was voluntary, and subjects were allowed to leave the testing area at any point throughout the session. Subjects' usual feeding and cleaning routines were followed, minimising disruption to the animals. The experimental phase of this study was ethically reviewed and approved by the University of Birmingham Animal Welfare and Ethical 
267 Review Body (UOB 31213) and by Twycross Zoo Research Committee (TZR-2017- 013),

268 following guidelines provided by SSSMZP, EAZA, BIAZA, WAZA on animal welfare and

269 research in zoological institutions; this study also received a letter of support from BIAZA. This

270 study adhered to legal requirements of the UK, where the research was carried out, and adhered

271 to the ASP principles for the Ethical Treatment of Primates.

272 Motivation tests

273 This phase took place between $13^{\text {th }}$ June 2017 and $27^{\text {th }}$ September. Prior to starting

274 experimental testing, it was important to ensure that the subjects were sufficiently interested and

275 motivated to access the novel food reward (macadamia nuts) used in this study. To motivate the

276 chimpanzees to try the nut kernels when first presented, the first stage involved a trusted

277 individual (a keeper that has worked with the subjects for more than five years) first eating a

278 different familiar food in front of the subjects (here we used dried raisins and berries). The

279 keeper attracted a subject's attention by calling their name, and then ate a single item of the

280 familiar food (i.e., one raisin) in view of them. This process was repeated until each individual

281 had observed the consumption in a group context. The subjects were then provided with the same

282 food and required to eat it before moving onto the next step. As this food was familiar, this

283 occurred in every case. The next stage was to introduce the novel food (macadamia nut kernels

284 already without their shells). The same keeper ate a single macadamia kernel in the same way as

285 with the familiar foods. Again, each individual was given a demonstration (sometimes groups of

286 individuals could watch together as subjects were not separated during this part of testing). Once

287 each individual had observed the consumption of the nuts at least once, they were provided with

288 a macadamia kernel, again within a group context. This process was designed to increase the

289 likelihood that the subjects would consume the novel food, as prior research has shown that

290 captive chimpanzees can vary substantially in their acceptance of novel food sources 
291 (Visalberghi, Yamakoshi, Hirata, \& Matsuzawa, 2002). Despite the neophobia reported by

292 Visalberghi et al. (2002), we chose to replicate their 'trusted' human demonstrator condition in

293 an attempt to maximise the likelihood that the subjects would consume the macadamia nuts. In

294 addition to this, the 'motivation tests' were used to ensure that the nuts were palatable to the

295 subjects; therefore, should they have succeeded in cracking a nut, they would be sufficiently

296 motivated to continue doing so. Equally, during the demonstration conditions (see below) the

297 nuts provided might then serve as a suitable motivator to encourage the chimpanzees to attempt

298 to reinnovate or copy the behaviour. We required at least half the chimpanzees to eat the novel

299 nuts before starting testing. This was to ensure that the motivation testing did not go on for too

300 long, as these tests were carried out within a group context. It was likely that lower ranking

301 individuals would never be allowed access to the nut kernels.

\section{Test conditions}

303 Each trial was video recorded, starting when the subjects were given access to the testing

304 apparatus. The study took place between the morning and afternoon feeds; this time was chosen

305 as it complemented the daily routine of the keepers and animals whilst providing the maximum

306 testing time possible. The timings changed once the chimpanzees moved enclosure as the

307 keepers were able to provide the afternoon feed without needing to move the subjects outside the

308 testing area. Average trial length before the move was 3 hours $(n=8)$, after the move it was 5

309 hours 41 minutes $(n=12)$. Overall, there was a total of 92 hours and 18 minutes observation

310 time $\left(\mathrm{M}_{\text {trial length }}=4\right.$ hours 37 minutes). The experimenter (DN) was present throughout each trial.

311 This study used a stepwise design, where each condition (see Figure 1) was followed by

312 the next in the event that the behaviour was not expressed in the first condition after five trials.

313 For example, the "End state" condition was only implemented in the event that the behaviour 
314 was not reinnovated in the "Baseline" condition. Testing ended once the subjects had received 5

315 trials with full action demonstrations.

316

317

318

319

320

321

322

323

324 325

326

328

329

330

331

332

333

334

335

336 337

\section{FIGURE 1 ABOUT HERE}

In all of the conditions, behaviours were first live coded. If, during live coding, any attempts at nut-cracking were identified then these were checked against videos and then second coded for reliability analysis. Here we defined nut-cracking in terms of tool use, therefore, to qualify as nut-cracking, the subject needed to use an object as a hammer to attempt to break open the nut, whilst resting the nut on another hard surface (the anvil). Video recordings were focussed on the experimental hammer and anvil set up (described below), however DN was present at all times to observe any behaviours that might have occurred outside of the camera frame.

\section{Materials}

The same apparatus set up was used in all conditions, and any changes to these conditions are noted in the relevant section. To set up the apparatus, DN entered the outdoor enclosure and secured a large wooden $\log (50 \mathrm{~cm}$ tall $\mathrm{x} 40 \mathrm{~cm}$ approx. diameter; that would serve as an "anvil") to an upright portion of the climbing frame (which had a horizontal crossbeam, to ensure that the anvil could not be removed; see Figure 2). The anvil was secured to the upright climbing frame using two $1 \mathrm{~m}$ long, $8 \mathrm{~mm}$ thick, PVC coated, steel rope passed through two (12mm diameter) holes drilled through the anvil (located $1 / 4$ and $3 / 4$ of the way down the log). Both ends of the ropes had a loop (secured by five 'clips' at each point, 'clips' used two, $8 \mathrm{~mm}$, nuts and bolts;tightened using an electric drill), which was too large to pass through the hole in the anvil, and a steel padlock attached the two ends. Two of these securing attachments were used as a failsafe measure (see Figure 2). 
A wooden "hammer" was also attached to this structure (wooden, rather than stone,

341 hammers were chosen as they were more secure in their attachment to the rope). The hammer

342 consisted of a $30 \mathrm{~cm}$ long x $15 \mathrm{~cm}$ diameter $\log$ - approx. weight $2.5 \mathrm{~kg}$ - with a $12 \mathrm{~mm}$ hole

343 drilled through half way along (see Figure 2). Hammer length was chosen based on the

344 descriptions of hammers used in wild populations to crack coula nuts $(20-80 \mathrm{~cm}$ long; Boesch \&

345 Boesch, 1983). Our diameter was chosen to be larger than these wild hammers $(4-10 \mathrm{~cm}$ in the

346 wild; Boesch \& Boesch, 1983) in the interest of safety being more likely to remain attached to

347 the securing attachment (see below). As a result of this increased diameter the hammers were

348 slightly heavier than the majority of those used in wild populations $(77 \%$ of which were less than

$3492 \mathrm{~kg}$; however, our hammers were still within the 2-4kg larger range described in wild

350 populations; Boesch \& Boesch, 1983).

351 The hammer was attached to the anvil's own securing attachment by creating a looped

352 end in another $(1.5 \mathrm{~m})$ length of the same steel rope; the loop was passed onto the top securing

353 attachment (of the anvil) and the loose end was secured to the hammer (by passing the loose end

354 through the drilled hole and then securing with another five clips). The hammer was then moved

355 less than $1 \mathrm{~m}$ from the anvil (see Figure 3 ).

356

357

FIGURE 3 ABOUT HERE

358

The keepers then scattered three macadamia nuts (in their shell) per individual (i.e., 3

360 nuts $\mathrm{x} 13$ individuals $=39$ nuts) throughout the enclosure, avoiding a $2 \mathrm{~m}$ radius around the 
361 hammer and anvil set-up. The macadamia nuts were distributed at the same time as a regular

362 scatter feed - just prior to the subjects being released into the outdoor enclosure. The unshelled

363 weight of the nuts (around $1 \mathrm{~g}$ average across 10 measurements) was taken from the

364 chimpanzees' usual allowance of nuts for the week (this was to maintain the dietary health of the

365 subjects, at the testing institution's request). Once the attachments had been checked by DN and

366 at least one keeper, all humans exited the enclosure and the chimpanzees were allowed in the

367 enclosure. Just prior to the chimpanzees being allowed access, video cameras (SONY HDR-

368 CX330e), set at two points framing the apparatus (to better capture various angles), on tripods,

369 were set to record. DN was also present to live code relevant behaviours (see Table 2) that

370 occurred outside of the frame of the fixed cameras.

\section{Baseline condition}

372 This test condition took place between $15^{\text {th }}$ October 2017 and $30^{\text {th }}$ November 2017.

373

374

375

376

377

378

379

380

381

382

383

384

\section{FIGURE 4 ABOUT HERE}

In order to examine whether the subjects would individually reinnovate the target nutcracking behaviour, it was necessary to test subjects without providing any social information beforehand. All sessions began between 10 am and 12 noon, when keepers provided the chimpanzees' scatter feed (mainly consisting of vegetables and fruit). All sessions were conducted in the "Outdoor 1" section of the enclosure (see Figure 4); however, subjects had access to both indoor areas throughout the session.

\section{End state condition}

This phase of the study was completed between $15^{\text {th }}$ January 2018 and $18^{\text {th }}$ May 2018. However, after the first two trials (15 $5^{\text {th }}$ January 2018 and $17^{\text {th }}$ January 2018) the weather 
385 conditions at the testing institution became so harsh that the subjects would often refuse to leave

386 the indoor enclosure. Thus, testing was paused until $14^{\text {th }}$ May 2018, after which the final three

387 trials were completed on the $14^{\text {th }}, 16^{\text {th }} \& 18^{\text {th }}$ May. Between testing in January and May subjects

388 were moved from "Conversion" (their previous enclosure) to a new enclosure: "Eden" (see

389 Figure 5); subjects were therefore given one month after moving to the new enclosure to settle in

390 before testing resumed.

391

392

FIGURE 5 ABOUT HERE

393

394

In this condition, we placed three macadamia nuts, shells and kernels, which had been

395

split in half (see Figure 6) on top of the anvil (in the "Conversion" enclosure this was in

"Outdoor 1" and in "Eden" this was in "Habitat 1"). This condition was designed to specifically

trigger stimulus/local enhancement (defined as when an animal's attention is drawn to an

object/location as a result of some change in the environment). In this condition, we drew a

400 the anvil.

FIGURE 6 ABOUT HERE

403

This condition was carried-out as the chimpanzees failed to individually reinnovate the nut-cracking behaviour in the baseline condition and followed the exact same protocol as the individual learning condition, described above. During the design process the study originally included an extra condition between the "Baseline" and "End state" conditions, called "Local 
408 Enhancement". In this condition it would have been made clear to the subjects that a kernel is

409 inside the macadamia nut and therefore that it constitutes a food source by shaving half of the nut

410 shell away to reveal the kernel inside (see Bandini and Tennie, 2018). However, some of the

411 chimpanzees in this study cracked the shells of the macadamia nuts with their teeth and

412 subsequently consumed the kernels (see Figure 7), rendering this condition unnecessary. This

413 was unexpected as Boesch and Boesch (1983) state that they never observed a wild chimpanzee

414 cracking coula nuts with its teeth; macadamia nuts (as used in this study) have a break strain of

415 between 1800-4000N (Schüler, Speck, Bührig-Polaczek, \& Fleck, 2014), which is comparable to

416 coula nuts and substantially less than required for panda nuts (Boesch, Bombjaková, Boyette, \&

417 Meier, 2017).

418

FIGURE 7 ABOUT HERE

420

421 Ghost condition

422

This phase of the study was completed between $19^{\text {th }}$ July 2018 and $10^{\text {th }}$ August 2018 . The

423 ghost condition involved a significant increase in the level of social information provided to the

424 subjects. In this condition, the hammer and anvil set-up were still present inside the enclosure, 425 along with three macadamia nuts per individual (scattered throughout "Eden Habitat 2") and a 426 further three nuts (this time whole and uncracked nuts, inside the shell was provided) placed on

427 top of the anvil. Additionally, a replica of the equipment inside the enclosure (i.e., a hammer and 428 anvil set up) was placed outside the enclosure, visible through the mesh near the subjects'

429 sleeping area (see Figures 8 \& 9); DN was also present, standing to the left of the anvil. 430 
434 hammer and passed through a section of mesh, allowing the hammer to be raised (between 80

435 and $50 \mathrm{~cm}$ ) above the anvil, via a pulley-like system (see Figure 9). A keeper steadied the

436 hammer before dropping it onto the nut, thus increasing the likelihood of the hammer cracking

437 the nut in the shortest possible time. Once the subject was clearly attending the apparatus (a 438 subject's attention was gained by calling their names), the hammer was dropped onto a nut

439 (which was placed in a groove in the centre of the anvil), cracking the nut open- this did not 440 always occur first time and may have required multiple attempts. A keeper then approached the 441 anvil and gave the subject who watched the demonstration the cracked nut. The device was then 442 rebaited with a new nut in the centre of the anvil. This procedure was repeated for a further 29 443 nuts (equalling a total of 30 demonstrations; with the exception of trial 4 , where the line broke, 444 meaning that the trial was halted after 17 demonstrations). Subjects had access to the testing 445 apparatus during the course of the ghost trials. One camera was used to record the subjects' 446 interactions with the test apparatus, whilst the other was used to record subjects observing the 447 ghost demonstrations; chest mounted GoPro (Hero5 Session) cameras were also used to record demonstrations and attention from the demonstrator's perspective. In both this condition and the

449 Full Action Demonstration condition, observing subjects were considered to be those in the 450 enclosure immediately in front of the demonstration area (far left sleeping area in Figure 8)

451 oriented towards the apparatus/demonstrator (i.e., not with their back turned). 452 
primary stipulation of learning by emulation (Tomasello et al., 1993); i.e., the learner should not

copy the motor patterns of the demonstrator. In this ghost condition, the motor patterns required

for nut-cracking were not demonstrated, making it impossible for the chimpanzees to copy the

inferred that the results of the actions were replicated rather than the actions themselves (Hopper,

461 2010; Whiten et al., 2004).

\section{Full action demonstration condition (human demonstrator)}

This phase of the study was completed between $16^{\text {th }}$ August 2018 and $6^{\text {th }}$ September

464 2018. The full action demonstration condition was the first one that allowed for the possibility of

465 action copying. In this condition, DN was positioned outside the enclosure (in the same location

466 as the ghost condition). An anvil was placed in the same location as in the ghost condition (see

467 Figure 8), with a hammer placed $1 \mathrm{~m}$ from the anvil (both pieces of wood were identical to those

468 in the subjects' enclosure). The researcher then attracted a subject's attention by calling their

469 name and proceeded to crack a nut, on top of the anvil, using the hammer. Note, it was not

470 possible to exclude the fact that multiple subjects may attend to the call of one individual -

471 subjects attending to a demonstration were coded from videos. The experimenter used the

472 hammer in a vertical manner, in the same orientation to the hammer in Figure 9 (see Figure 10),

473 raising it to eye level and then hitting down onto the nut, resulting in the nut breaking open. The

474 orientation of the hammer was used to attempt to control for hammer orientation between the

475 ghost and full action demonstration trials. Once cracked, the kernel was provided to the subject

476 by a keeper (see Figure 10D) and the device rebaited with another nut. A total of 30 nuts were

477 cracked using this procedure in each trial; a nut was not cracked until DN considered that the

478 target subject was attending to the demonstration. A maximum of 30 nuts was used based on 
479 advice from keepers that not all subjects would attend to, or even approach, the demonstrations

480 (therefore, for all subjects to observe, trials could have continued indefinitely which would have 481 been unfeasible).

482

483

FIGURE 10 ABOUT HERE

484

485 Coding/analysis of behaviours

486 Coding procedure

487 Trials were live coded using the ethogram in Table 2. Following live coding a formal 488 coding procedure from video was followed. DN coded each trial in turn and a second coder

489 (MT), naïve to the hypothesis of this study, second coded $100 \%$ of the behaviours identified $490(\mathrm{~N}=31)$ along with an equal number of "dummy" clips where a subject was in the frame but DN

491 did not identify a behaviour occurring to test for inter-rater reliability (acceptable Kappa would 492 be 0.6; Cohen, 1968; calculted using R package "irr" v.0.84.1; Gamer, Lemon, Fellows, \& Singh, 493 2019). Note that the behaviours in Table 2 rely on the previous behaviour in order for them to be 494 coded; e.g., if the subject picked up the hammer without first placing a nut on the anvil then the 495 hammer behaviour would not be coded. This was to attempt to parse hammer centred 496 play/exploration from attempts at nut-cracking.

497

498

TABLE 2 ABOUT HERE

499

500 Analyses

501 After a behaviour has been reinnovated, social facilitation cannot be excluded as a

502 potential reason for the behaviour's continued emergence in other group members (Bandini \&

503 Tennie, 2018; Tennie \& Hedwig, 2009). Given an $\mathrm{N}$ of 1, it is not possible to perform inferential 
504 statistics on acquisition times or rates between individuals. However, descriptive statistics were

505 used. All descriptive statistics were produced using R v.3.5.2 (R Core Team, 2013).

506 Results

507 Motivation test

508 During the motivation test, seven subjects (54\% of sample; C3, C7, C9, C8, C5, C12 \&

$509 \mathrm{C} 13$ ) consumed at least one macadamia nut provided by the keeper, leading to the conclusion

510 that macadamia nuts were/are palatable to most of subjects included in this study (although note

511 that dominance hierarchies/individual personality characteristics may have interfered with

512 certain individuals' ability/motivation to access the nuts).

\section{Reliability analysis}

514 The results of a Cohen's Kappa analysis revealed a strong level of agreement between

515 coders $(\kappa=.85, \mathrm{p}<.001)$.

516 Attempts at nut-cracking

517 None of the individuals in this study attempted to crack open the nuts using a tool in any

518 of the conditions described above. As there was never any evidence of nut-cracking, or

519 approximations of it, all conditions were completed (as explained in the methods section).

520 Attempts recorded within the ethogram

521 The coding procedure identified the following behaviours from the ethogram: place $(n=$

522 26; first occurring during baseline condition trial 2 but distributed across baseline $(n=7)$, end

523 state $(n=15)$ and ghost $(n=4)$ conditions), hold $(n=1$; occurring during baseline condition trial

5242 ), stamp ( $n=2$; occurring during baseline condition trial 2$)$ and throw $(n=2$; occurring during

525 baseline condition trial 2). Recordings of "place" were identified in C5 ( $n=7)$, C6 ( $n=18)$ and

526 C7 $(n=1)$ across all conditions apart from full demonstration. In only one instance did a "hold"

527 event follow "place", this concerned C6 during baseline condition trial 2; who was also the only

528 individual to "stamp" on or "throw" the nuts. It is unclear whether throwing was an active effort 
529 to break the nut or simply an act of frustration/play as it did not appear that the throws were

530 aimed at any hard surface, nor were there ever attempts to retrieve the nuts afterwards by the

531 throwers.

532 Alternative techniques

533 Anecdotally, the majority of subjects (if not all) were witnessed, at least once, attempting

534 to crack the nuts with their teeth (with some individuals succeeding; see Figure 7). Male

535 chimpanzees $(n=4)$ were the only individuals observed (by DN) successfully accessing the nut

536 kernel using this method. The teeth cracking technique was first observed in the baseline

537 condition and persisted throughout the study. These behaviours were not captured on the main

538 videos as the cameras were facing the apparatus throughout the trial (to ensure that any attempts

539 at using the apparatus to crack the nuts were captured), also some subjects were not visible

540 throughout; therefore, any attempt to quantify these behaviours would be inaccurate as it would

541 likely present only part of the actual series of events.

542 Observers in Ghost and Full Action Demonstration Conditions

543 Occasionally the identity of the observer could not be ascertained from video footage; in

544 these cases, the individuals were not included in the calculations below. Furthermore, as

545 participation in the study was voluntary, and subjects were free to approach and interact with the

546 testing apparatus whenever they chose, not all subjects observed all the demonstrations provided.

547 Some subjects $(n=2 ; \mathrm{C} 5 \& \mathrm{C} 11)$ never observed the demonstrations in either condition; whilst

548 other subjects never observed demonstrations in either the ghost $(n=3 ; \mathrm{C} 5, \mathrm{C} 13$ \& $\mathrm{C} 11)$ or full

549 demonstration $(n=4 ; \mathrm{C} 5, \mathrm{C} 11, \mathrm{C} 1, \mathrm{C} 10)$ conditions. Overall, $77 \%$ of subjects $(n=10)$ were

550 coded as observers in the ghost condition and $69 \%$ of subjects $(n=9)$ were coded as observers the

551 full demonstration condition; there was an average of 2.48 observers per ghost demonstration

552 and 2.99 observers per full demonstration. 


\section{Discussion}

554 We found no evidence of nut-cracking with a tool, or any approximation at this, at any

555 point during the course of this study. Thus, our sample of 13 naïve chimpanzees failed to

556 reinnovate or socially learn the behavioural form of nut-cracking. At first, it would seem our

557 findings support the CDT hypothesis, in that nut-cracking behaviour was not reinnovated in our

558 initial baseline condition. However, our study also consisted of various social learning test

559 conditions - including one that demonstrated the necessary action patterns for nut-cracking to the

560 chimpanzees. This condition allowed for the possibility of action copying being a requirement of

561 the behaviour, as has recently been claimed (Estienne, Cohen, Wittig, \& Boesch, 2019). Even so,

562 nut-cracking was not acquired by the subjects. Therefore, our study does not provide conclusive

563 evidence for either the CDT or the ZLS hypothesis. Below we discuss three possible

564 explanations for our null result and the general disparity in studies of chimpanzee nut-cracking.

565 Conspecific models

The findings of this study raise the question as to why some chimpanzee populations in

567 the wild regularly crack nuts (on average 270 nuts per day for as long as 2 hours 15 minutes in

568 Taï Forest; Boesch \& Boesch-Achermann, 2000) whereas captive chimpanzees (in this sample

569 and others; Funk, 1985) seem to rather consistently fail to acquire the behaviour, even after

570 demonstrations. A first possibility for the disparity between wild and captive data is that nut-

571 cracking is indeed a CDT and requires the learner to imitate a conspecific demonstrator (Boesch,

572 1996). In this study we used human demonstrators, which may not have been considered 'good'

573 enough models for the chimpanzees. Indeed, some research has shown that chimpanzees are

574 more proficient social learners from conspecific models as compared to videos or human models

575 (Hopper, Lambeth, Schapiro, \& Whiten, 2015). However, in contrast, others have instead

576 claimed that it is possible for chimpanzees to "learn" nut-cracking from human demonstrators 
577 (see findings of Ross, Milstein, Calcutt, \& Lonsdorf, 2010 but also review of other related

578 studies Table 3 (p. 230) of Ross et al. 2010). Concurrently, other studies have found that, even

579 with conspecific demonstrators, sometimes captive chimpanzees fail to acquire complex

580 behaviours such as nut-cracking (Funk, 1985) or behaviours which strictly require imitation

581 (Clay \& Tennie, 2018; Tennie, Call, \& Tomasello, 2012; Tomasello et al., 1997). Although it

582 might have been interesting to observe the chimpanzees' reaction to a conspecific demonstrator

583 in this study, we did not have the resources to train a chimpanzee to act as a demonstrator, but

584 we encourage interested researchers who do have the resources to replicate this study, and

585 include a conspecific demonstrator to observe whether this affects the findings presented here.

\section{Behavioural flexibility}

587 An alternative explanation for the fact that nut-cracking did not emerge in this study is

588 that the chimpanzees were hindered by their lack of behavioural flexibility, a commonly

589 recorded phenomenon in chimpanzees (e.g., Harrison \& Whiten, 2018). The chimpanzees in the

590 current study seemed to become fixated on one solution to open the nuts: i.e., the use of their

591 teeth. The chimpanzees may have relied on this technique due to their pre-existing knowledge on

592 how to crack softer-shelled nuts (such as peanuts and walnuts), which they are often provided

593 during their feeds at the testing institution. These nuts are easily cracked open by apes using

594 teeth (DN; personal observation, keeper reports and see also Visalberghi et al., 2008 for

595 measurements on the required force for different types of nuts). The heuristic (Marsh, 2002) in

596 this case may be that nuts (in general) can be opened with teeth - and indeed our macadamia

597 nuts were no exception. Chimpanzees have been shown to be reluctant to display behavioural

598 flexibility in abandoning a previously successful solution (see Harrison \& Whiten, 2018;

599 Hrubesch, Preuschoft, \& van Schaik, 2009; but see also Manrique, Völter, \& Call, 2013). Thus,

600 it is possible that the first individual to successfully crack a nut with the use of teeth (see Figure 
601 7), facilitated this behaviour within the group and/or that other individuals independently

602 converged on this method, and then the subjects were unable to innovate a new method, even if

603 cracking the nuts with a tool would have been mechanistically easier/more efficient (this

604 possibility is also in line with cultural founder effects; Tennie et al., 2009).

\section{Sensitive learning period}

606 Based on the literature, the most likely explanation for the findings in this study is that

607 the chimpanzees may have simply been outside of their sensitive learning period for nut-

608 cracking. Previous studies on nut-cracking in wild chimpanzees have reported that before 3.5

609 years, juvenile chimpanzees are unable to express the full nut-cracking behavioural form (Inoue-

610 Nakamura \& Matsuzawa, 1997; Matsuzawa, 1994). However, juvenile chimpanzees (as young as

611 1.5 years old) that had been exposed to the materials required for nut-cracking at various

612 ages/developmental stages were able to perform the basic actions of the behaviour (put, hold, hit

613 and eat), but not combine them in the required order to perform the full nut-cracking behaviour

614 (Inoue-Nakamura \& Matsuzawa, 1997). Indeed, recent research in wild chimpanzees shows an

615 exponential increase in nut-cracking between 5 and 6 years of age, though the first signs were

616 observed in 3-4-year-old individuals (Estienne et al., 2019) in line with the concept of maturation

617 (Corp \& Byrne, 2002). This finding suggests a certain level of developmental prowess required

618 to express nut-cracking, perhaps somewhere between maturation effects of the body and the

619 brain.

620

In addition to this lower age limit for the acquisition of nut-cracking, there also appears to

621 be an upper limit (more relevant for the current study). A 13 year longitudinal study by Biro et

622 al., (2003) found that wild chimpanzees who did not learn the basic nut-cracking skills before

623 five years old seemed unable to acquire the behaviour later on in adulthood (a similar case has

624 been documented recently for stone tool-use in long-tailed macaques; Tan, 2017). The subjects 
625 tested in the current study were all outside of the hypothesised sensitive learning period for nut-

626 cracking, as the youngest subject in our sample was already 10 years old at the time of testing.

627 The youngest individual however was the only subject to display the "hold" behaviour (stage two 628 of four) in the behavioural form of nut-cracking. Our findings, coupled with those described here 629 suggest that a sensitive learning period may be a decisive factor for whether a chimpanzee will 630 start to crack nuts or not (leaving open the question how this is learned, i.e. whether it is a CDT 631 or a latent solution).

632 Given the fact that wild chimpanzees engage in an extended process of acquisition before 633 expressing nut-cracking (Matsuzawa et al., 2008), we suggest that future work considers 634 applying an even longer study time than the one employed here. It is possible that chimpanzees 635 may then individually, or socially, learn the behaviour. The social learning oppertunities here 636 provided comparatively more demonstrations than related studies (5 trials with 30 nuts per 637 demonstration in our study versus 5 trials with 5 nuts per demonstration by Marshall-Pescini \&

638 Whiten; 2008). Even so, the social learning opportunities that we present here were fewer

639 compared to the wild; in wild populations that express the behaviour, individuals have more and 640 longer (and perhaps also more varied) opportunities to observe nut-cracking. Equally, given the 641 potential importance of a sensitive learning period in explaining the emergence patterns of 642 chimpanzee nut-cracking (discussed in this section) we suggest that the next logical test of this 643 behaviour should aim to test younger chimpanzees between the ages of 3 and 10 years (Ross et 644 al., 2010 suggest between ages 3-7); either way, these individuals should once again be selected 645 from populations that have not been observed previously to crack nuts.

646 Though we used a within-subject design throughout our result-dependent design, we 647 would reccomend that (wherever feasible) a between-subjects design be used in future tests (one 
648 group for each of the conditions). By doing the latter, it is possible to control for and measure the

649 time of exposure required for chimpanzees to express nut-cracking, and it would exclude

650 potential carry-over effects. However, this project would likely be an overly large undertaking

651 for any one research group, so therefore may be better suited to large scale collaborative projects

652 (e.g., the ManyPrimates project).

\section{Conclusions}

654 Although no chimpanzees in this study demonstrated nut-cracking using tools, two

655 geographically separate populations in the wild have seemingly converged on the same method

656 for cracking nuts using tools (West Africa; Whiten et al., 2001 and Cameroon; Morgan \& Abwe,

657 2006). As these populations do not have access to each other, logically they must have

658 independently reinnovated nut-cracking (Byrne, 2007). However, the data from Cameroon is

659 based on procured nut cracking tools and auditory assumptions and so are an inference rather

660 than direct observations. It is possible that these tools were actually from modern human nut-

661 crackers and may not be considered evidence of chimpanzee nut-cracking in multiple

662 populations. Thus, to date, there is only concrete evidence of one culturally independent wild

663 population expressing nut-cracking. As the data from this study and wild data do not

664 unequivocally support either the ZLS or the CDT hypothesis, both remain in contention.

665 In addition to the wild data and that presented here, one chimpanzee in an experimental

666 study spontaneously reinnovated nut-cracking when provided with all the materials (Marshall-

667 Pescini \& Whiten, 2008). The individual (Mawa) acquired the nut-cracking behaviour seemingly

668 without requiring any copying variants of social learning (Marshall-Pescini \& Whiten, 2008).

669 However, Marshall-Pescini \& Whiten (2008) fail to note the importance of these findings, by

670 assuming, based on the speed of acquisition, that "Mawa" had prior experience of nut-cracking.

671 It is worthy of note however that unlike this study and that of Funk (1985), no claim was made 
672 about the naivety of the subjects. Indeed, Mawa was kept as a pet prior to residing at the

673 sanctuary where Marshall-Pescini and Whiten (2008) carried-out their study. Mawa arrived at

674 the sanctuary when he was approx. three years old with wounds from a rope where he was tied

675 up (Ferdowsian et al., 2011). As a result of this potential enculturation, or at the very least

676 deprivation, these data should be treated with caution; indeed, the generalisability of such

677 individuals to wild chimpanzees is questionable (Henrich \& Tennie, 2017).

678 Excavations of chimpanzee nut-cracking sites suggest that the basic behavioural form

679 has remained constant for at least 4,000 years, and likely even longer (Mercader et al., 2007).

680 Similarly, excavations of capuchin nut-cracking sites have demonstrated that their nut-cracking

681 form has remained the same for 3,000 years, with only the tools (hammerstones) changing in

682 shape over time (Falótico, Proffitt, Ottoni, Staff, \& Haslam, 2019). Indeed, if the behavioural

683 form of nut-cracking were being copied between individuals, we would expect to see some

684 changes to its form over time due to copying error alone (see Eerkens \& Lipo, 2005). Lastly,

685 other extant species of primates also crack nuts using tools in the wild (long tailed macaques,

686 Gumert \& Malaivijitnond, 2013; capuchins, Ottoni \& Mannu, 2001), and some have even been

687 found to do so spontaneously in captivity without requiring social learning (e.g., nut-cracking is

688 a latent solution in capuchins: Visalberghi, 1987). Whilst other primate species being able to

689 spontaneously crack nuts is not evidence of a phylogenetic link; it does suggest that the

690 possibility that nut-cracking is a latent solution in chimpanzees remains. Therefore, social

691 learning may not be fully responsible for the emergence of nut-cracking in chimpanzees. We

692 acknowledge that the chimpanzees in this study were captive and therefore are not subject to the

693 same ecological pressures as their wild conspecifics; that is, they would have less 'necessity' to 
694 reinnovate the behaviour (Fox, Sitompul \& van Schaik, 1999). Therefore, (parts of) this study

695 could perhaps be replicated in a wild sample, naïve to nut-cracking.

696 Therefore, the results of this study do not support nut-cracking as the first evidence of a

697 CDT in chimpanzees (see also Byrne, 2007), yet they also do not support nut-cracking as a latent

698 solution in chimpanzees. Instead, we conclude that the behaviour may not have emerged here

699 due to interplay of factors, including a certain level of behavioural conservatism and, crucially,

700 the fact that all the subjects were already out of their sensitive learning periods for nut-cracking.

701 We believe it is unlikely that our use of human demonstrators was the reason for the failure of all

702 our subjects to express nut-cracking, given the results of previous studies, discussed above.

703 Accordingly, we propose that future studies should adopt the methodology presented here, but

704 test unenculturated infant/juvenile chimpanzees, naïve to nut-cracking and to opening nuts with

705 their teeth, to remove the confounds of the sensitive learning periods and conservatism (ideally

706 tested in isolation in order to increase effective sample size). Under these conditions, it is

707 plausible that some naïve chimpanzees will reinnovate nut-cracking. Yet, on the other hand,

708 given the extended trial-and-error learning process that young wild chimpanzees engage in

709 (Matsuzawa et al., 2008) it is possible that under the relatively short term test conditions, the full

710 form of nut-cracking may still fail to emerge spontaneously, although some of the pre-requisite

711 steps to the behaviours may still develop.

712 So far, the current state of knowledge does not support the view that nut-cracking has to

713 be reliant on social learning as it has potentially been reinnovated in two culturally distinct

714 populations, therefore, it seems unlikely that it is a CDT. However, it is also possible that

715 chimpanzees within their sensitive learning period may fail to individually acquire the skills

716 required to crack nuts; in this case nut-cracking could not be considered a CDT. The data at hand 
717 suggest that the behavioural form of nut-cracking may only be acquired through an interplay of

718 ecological and developmental factors, i.e., chimpanzees must be in a location with appropriate

719 nuts and tool materials, during or before, their sensitive learning period. Therefore, it remains

720 possible that nut-cracking is within the species level ZLS of chimpanzees. Despite this, not all

721 individuals may realise this potential within their lifetime if they were not exposed to the

722 required ecological conditions or individual prerequisites (note that these were termed by Tennie

723 et al. (2009) as the "right" conditions that may be required). It is yet to be determined whether

724 nut-cracking's acquisition is best described as being due to, and requiring, social learning

725 (culture-dependent) or is due to socially mediated reinnovation (latent solution). Further research

726 should consider the importance of the ecological factors explored here in addressing this

727 question.

728

729

730

731

732

733

734

735

736

737

738

\section{Acknowledgements}

We would like to acknowledge the support of Twycross Zoo throughout the process of this study, without their support in material acquisition and access to subjects this study would not have been possible. In particular we would like to thank Clare Ellis, Freisha Patel, Katie Waller, Kris Hern, the entire ape team and the staff at Wates for their support. In addition, we would like to thank Jackie Chappell, Sarah Beck, Susannah Thorpe and Josep Call for their helpful discussions regarding this study. We would also like to thank Lydia Hopper, Lydia Luncz and two other anonymous reviewers for helpful comments on an earlier version of this manuscript. Finally, we would like to thank Matthew Thompson for his help in completing second coding for reliability analyses. 


\section{References}

740 Bandini, E., \& Tennie, C. (2017). Spontaneous reoccurrence of “scooping”, a wild tool-use

741 behaviour, in naïve chimpanzees. PeerJ, 5(e3814). https://doi.org/10.7717/peerj.3814

742 Bandini, E., \& Tennie, C. (2018). Naive, captive long-tailed macaques (Macaca fascicularis

743 fascicularis) fail to individually and socially learn pound-hammering, a tool-use behaviour.

744 Royal Society Open Science, 5(5). https://doi.org/10.1098/rsos.171826

745 Bandini, E., \& Tennie, C. (2019). Individual acquisition of "stick pounding" behavior by naïve

746 chimpanzees. American Journal of Primatology, e22987. https://doi.org/10.1002/ajp.22987

747 Biro, D., Inoue-Nakamura, N., Tonooka, R., Yamakoshi, G., Sousa, C., \& Matsuzawa, T. (2003).

748 Cultural innovation and transmission of tool use in wild chimpanzees: evidence from field

749 experiments. Animal Cognition, 6, 213-223. https://doi.org/10.1007/s10071-003-0183-x

750 Boesch, C. (1996). Three approaches to investigating chimpanzee culture. In A. E. Russon, K. A.

751 Bard, \& S. T. Parker (Eds.), Reaching Into Thought: The Minds of the Great Apes (pp. 404-

752 429). Cambridge, UK: Cambridge University Press.

753 Boesch, C., \& Boesch-Achermann, H. (2000). Chimpanzees of the Taï Forest: Behavioural

754 Ecology and Evolution. Oxford, UK: Oxford University Press.

755 Boesch, C., \& Boesch, H. (1983). Optimisation of nut-cracking with natural hammers by wild

756 chimpanzees. Behaviour, 83(3-4), 265-286. https://doi.org/10.1163/156853983X00192

757 Boesch, C., \& Boesch, H. (1984). Mental map in wild chimpanzees: An analysis of hammer

758 transports for nut cracking. Primates, 25(2), 160-170. https://doi.org/10.1007/BF02382388

759 Boesch, C., Bombjaková, D., Meier, A., \& Mundry, R. (2019). Learning curves and teaching

760 when acquiring nut-cracking in humans and chimpanzees. Scientific Reports, 9(1), 1515.

$761 \quad$ https://doi.org/10.1038/s41598-018-38392-8

762 Boyd, R., \& Richerson, P. J. (1996). Why culture is common, but cultural evolution is rare. 
763

764

765

766

767

768

769

770

771

772

773

774

775

776

777

778

779

780

781

782

783

784

785

Proceedings of the British Academy, 88, 77-93. Retrieved from

http://cat.inist.fr/?aModele=afficheN\&cpsidt=2887195

Byrne, R. W. (2007). Culture in great apes : using intricate complexity in feeding skills to trace the evolutionary origin of human technical prowess. Philosophical Transactions of the Royal Society B, 362, 577-585. https://doi.org/10.1098/rstb.2006.1996

Byrne, R. W., Corp, N., \& Byrne, J. M. E. (2001). Estimating the complexity of animal behaviour: how mountain gorillas eat thistles. Behaviour, 138, 525-557. https://doi.org/10.1163/156853901750382142

Caldwell, C. A., \& Millen, A. E. (2009). Social learning mechanisms and cumulative cultural evolution: is imitation necessary?. Psychological Science, 20(12), 1478-1483. https://doi.org/10.1111/j.1467-9280.2009.02469.x

Clay, Z., \& Tennie, C. (2018). Is overimitation a uniquely human phenomenon? Insights from human children as compared to bonobos. Child Development, 89(5), 1535-1544. https://doi.org/10.1111/cdev.12857

Cohen, J. (1968). Weighted kappa: nominal scale agreement with provision for scaled disagreement or partial credit. Psychological Bulletin, 70(4), 213-220. https://doi.org/10.1037/h0026256

Collias, N. E., \& Collias, E. C. (1984). Nest Building and Bird Behavior. Princeton, NJ: Princeton University Press.

Corp, N., \& Byrne, R. W. (2002). The ontogeny of manual skill in wild chimpanzees: evidence from feeding on the fruit of Saba florida. Behaviour, 139, 137-168. https://doi.org/10.1163/15685390252902328

Dean, L. G., Vale, G. L., Laland, K. N., Flynn, E., \& Kendal, R. L. (2014). Human cumulative 
culture: a comparative perspective. Biological Reviews, 89(2), 284-301. https://doi.org/10.1111/brv.12053

788

789

790

791

792

793

794

795

796

797

798

799

800

801

802

803

804

805

806

807

808

Eerkens, J. W., \& Lipo, C. P. (2005). Cultural transmission, copying errors, and the generation of variation in material culture and the archaeological record. Journal of Anthropological Archaeology, 24(4), 316-334. https://doi.org/10.1016/j.jaa.2005.08.001

Estienne, V., Cohen, H., Wittig, R. M., \& Boesch, C. (2019). Maternal influence on the development of nut-cracking skills in the chimpanzees of the Taï forest, Côte d'Ivoire ( Pan troglodytes verus ). American Journal of Primatology, 81(7), e23022. https://doi.org/10.1002/ajp.23022

Falótico, T., Proffitt, T., Ottoni, E. B., Staff, R. A., \& Haslam, M. (2019). Three thousand years of wild capuchin stone tool use. Nature Ecology \& Evolution, 3, 1034-1038. https://doi.org/10.1038/s41559-019-0904-4

Ferdowsian, H. R., Durham, D. L., Kimwele, C., Kranendonk, G., Otali, E., Akugizibwe, T., ... Johnson, C. M. (2011). Signs of mood and anxiety disorders in chimpanzees. PLoS ONE, 6(6), e19855. https://doi.org/10.1371/journal.pone.0019855

Foucart, J., Bril, B., Hirata, S., Monimura, N., Houki, C., Ueno, Y., \& Matsuzawa, T. (2005). A preliminary analysys of nut-cracking movements in a captive chimpanzee: adaptation to the properties of tools and nuts. In V. Roux \& B. Brill (Eds.), Stone Knapping: The Necessary Conditions for a Uniquely Hominin Behaviour (pp. 147-157). Cambridge, UK: McDonald Institute for Archaeological Research.

Fox EA, Sitompul AF, van Schaik CP. (1999). Intelligent tool use in Sumatran orangutans. In Parker ST, Mitchell RW, Miles HL (Eds.), The mentalities of gorillas and orangutans (pp. 99-116). Cambridge, UK: Cambridge University Press. 
809 Funk, M. (1985). Werkzeuggebrauch Beim öffnen von Niessen: Unterschiedliche Bewaltigungen

810 des Problems bei Schimpansen und Orang-Utans. University of Zurich.

811 Galef, B. G. (1992). The question of animal culture. Human Nature, 3(2), 157-178.

812 Gamer, M., Lemon, J., Fellows, I., \& Singh, P. (2019). Various coefficients of interrater

813 reliability and agreement. CRAN. Retrieved from https://cran.r-

$814 \quad$ project.org/web/packages/irr/index.html

815 Goren-Inbar, N., Sharon, G., Melamed, Y., \& Kislev, M. (2002). Nuts, nut cracking, and pitted

816 stones at Gesher Benot Ya'aqov, Israel. Proceedings of the National Academy of Sciences,

817 99(4), 2455-2460. https://doi.org/10.1073/pnas.032570499

818 Gumert, M. D., \& Malaivijitnond, S. (2013). Long-tailed macaques select mass of stone tools

819 according to food type. Philosophical Transactions of the Royal Society B: Biological

$820 \quad$ Sciences, 368(1630). https://doi.org/10.1098/rstb.2012.0413

821 Haidle, M. N. (2012). A Comparison of Cognitive Aspects in Tool Behavior of Animals and

822 During Human Evolution. Cognitive Perspectives in Tool Behaviour. Retrieved from

823 http://tobias-lib.uni-tuebingen.de/frontdoor.php?source_opus=6014

824 Harrison, R. A., \& Whiten, A. (2018). Chimpanzees (Pan troglodytes) display limited

825 behavioural flexibility when faced with a changing foraging task requiring tool use. PeerJ,

$826 \quad 6$, e4366. https://doi.org/10.7717/peerj.4366

827 Haslam, M. (2014). On the tool use behavior of the bonobo-chimpanzee last common ancestor, 828 and the origins of hominine stone tool use. American Journal of Primatology, 76(10), 910-

829 918. https://doi.org/10.1002/ajp.22284

830 Hayashi, M., Mizuno, Y., \& Matsuzawa, T. (2005). How does stone-tool use emerge?

831 Introduction of stones and nuts to naïve chimpanzees in captivity. Primates, 46(2), 91-102. 
833 Henrich, J., \& Tennie, C. (2017). Cultural evolution in chimpanzees and humans. In M. Muller,

834 R. W. Wrangham, \& D. Pilbeam (Eds.), Chimpanzees and Human Evolution (pp. 645-702).

835 Cambridge, MA: Harvard University Press.

836 Heyes, C. (1994). Social learning in animals: categories and mechanisms. Biological Reviews, 837 69, 207-231. https://doi.org/10.1111/j.1469-185X.1994.tb01506.x

838 Hopper, L. M. (2010). "Ghost" experiments and the dissection of social learning in humans and

839 animals. Biological Reviews, 85(4), 685-701. https://doi.org/10.1111/j.1469-

$840 \quad 185 X .2010 .00120 . x$

841 Hopper, L. M., Lambeth, S. P., Schapiro, S. J., \& Whiten, A. (2008). Observational learning in

842 chimpanzees and children studied through "ghost" conditions. Proceedings. Biological

843 Sciences / The Royal Society, 275(1636), 835-840. https://doi.org/10.1098/rspb.2007.1542

844 Hopper, L. M., Lambeth, S. P., Schapiro, S. J., \& Whiten, A. (2015). The importance of

845 witnessed agency in chimpanzee social learning of tool use. Behavioural Processes, 112,

846 120-129. https://doi.org/10.1016/j.beproc.2014.10.009

847 Hrubesch, C., Preuschoft, S., \& van Schaik, C. P. (2008). Skill mastery inhibits adoption of

848 observed alternative solutions among chimpanzees (Pan troglodytes). Animal Cognition, 12,

849 209-216. https://doi.org/10.1007/s10071-008-0183-y

850 Inoue-Nakamura, N., \& Matsuzawa, T. (1997). Development of stone tool use by wild

851 chimpanzees (Pan troglodytes). Journal of Comparative Psychology, 111(2), 159-173.

$852 \quad$ https://doi.org/10.1037/0735-7036.111.2.159

853 Kendal, R. L. (2008). Animal “culture wars.” The Psychologist, 21(4), 312-315. Retrieved from

854 https://thepsychologist.bps.org.uk/volume-21/edition-4/animal-culture-wars 
855 Luncz, L. V., Mundry, R., \& Boesch, C. (2012). Evidence for cultural differences between

856 neighboring chimpanzee communities. Current Biology, 22(10), 922-926.

857 https://doi.org/10.1016/j.cub.2012.03.031

858 Lycett, S. J., Collard, M., \& McGrew, W. C. (2007). Phylogenetic analyses of behavior support

859 existence of culture among wild chimpanzees. Proceedings of the National Academy of

$860 \quad$ Sciences of the United States of America, 104(45), 17588-17592.

$861 \quad$ https://doi.org/10.1073/pnas.0707930104

862 Lycett, S. J., Collard, M., \& McGrew, W. C. (2010). Are behavioral differences among wild

863 chimpanzee communities genetic or cultural? An assessment using tool-use data and

864 phylogenetic methods. American Journal of Physical Anthropology, 142(3), 461-467.

865 https://doi.org/10.1002/ajpa.21249

866 Manrique, H. M., Völter, C. J., \& Call, J. (2013). Repeated innovation in great apes. Animal

867 Behaviour, 85(1), 195-202. https://doi.org/10.1016/j.anbehav.2012.10.026

868 Marsh, B. (2002). Do animals use heuristics? Journal of Bioeconomics, 4, 49-56.

869 https://doi.org/10.1023/A:1020655022163

870 Marshall-Pescini, S., \& Whiten, A. (2008). Social learning of nut-cracking behavior in East

871 African sanctuary-living chimpanzees (Pan troglodytes schweinfurthii). Journal of

872 Comparative Psychology, 122(2), 186-194. https://doi.org/10.1037/0735-7036.122.2.186

873 Matsuzawa, T. (1994). Field experiments on use of stone tools by chimpanzees in the wild. In R.

874 W. Wrangham, W. C. McGrew, F. B. M. de Waal, \& P. G. Heltne (Eds.), Chimpanzee

875 Cultures (pp. 351-370). Cambridge, MA: Harvard University Press.

876 Matsuzawa, T., Biro, D., Humle, T., Inoue-Nakamura, N., Tonooka, R., \& Yamakoshi, G.

877 (2008). Emergence of culture in wild chimpanzees: education by master-apprenticeship. In 
T. Matsuzawa (Ed.), Primate Origins of Human Cognition and Behavior (pp. 557-574).

879 Tokyo: Springer.

880 McGrew, W. C., Ham, R. M., White, L. J. T., Tutin, C. E. G., \& Fernandez, M. (1997). Why

881 don't chimpanzees in Gabon crack nuts? International Journal of Primatology, 18(3), 353-

882 374. https://doi.org/10.1023/A:1026382316131

883 Menzel, C., Fowler, A., Tennie, C., \& Call, J. (2013). Leaf surface roughness elicits leaf

884 swallowing behavior in captive chimpanzees (Pan troglodytes) and bonobos (P. paniscus),

885 but not in gorillas (Gorilla gorilla) or orangutans (Pongo abelii). International Journal of

886 Primatology, 34(3), 533-553. https://doi.org/10.1007/s10764-013-9679-7

887 Mercader, J., Barton, H., Gillespie, J., Harris, J., Kuhn, S., Tyler, R., \& Boesch, C. (2007).

8884,300 -year-old chimpanzee sites and the origins of percussive stone technology.

889 Proceedings of the National Academy of Sciences, 104(9), 3043-3048.

$890 \quad$ https://doi.org/10.1073/pnas.0607909104

891 Miton, H., \& Charbonneau, M. (2018). Cumulative culture in the laboratory: Methodological and

892 theoretical challenges. Proceedings of the Royal Society B: Biological Sciences, 285(1879),

893 20180677. https://doi.org/10.1098/rspb.2018.0677

894 Morgan, B. J., \& Abwe, E. E. (2006). Chimpanzees use stone hammers in Cameroon. Current

895 Biology, 16(16), 632-633. https://doi.org/10.1016/j.cub.2006.07.045

896 Motes-Rodrigo, A., Majlesi, P., Pickering, T. R., Laska, M., Axelsen, H., Minchin, T. C., ...

897 Adriana Hernandez-Aguilar, R. (2019). Chimpanzee extractive foraging with excavating

898 tools: experimental modeling of the origins of human technology. PLoS ONE, 14(5), 12-16.

899 https://doi.org/10.1371/journal.pone.0215644

900 Muthukrishna, M., \& Henrich, J. (2016). Innovation in the collective brain. Philosophical 
901

902

903

904

905

906

907

908

909

910

911

912

913

914

915

916

917

918

919

920

921

922

923

Transactions of the Royal Society B: Biological Sciences, 371(1690), 20150192. https://doi.org/10.1098/rstb.2015.0192

Neadle, D., Allritz, M., \& Tennie, C. (2017). Food cleaning in gorillas: social learning is a possibility but not a necessity. PLoS ONE, 12(12), e0188866. https://doi.org/10.1371/journal.pone.0188866

Odling-Smee, F. J., Laland, K. N., \& Feldman, M. W. (2003). Niche Construction: The neglected process in evolution. Princeton: Princeton University Press.

Oswalt, W. H. (1976). An Anthropological Analysis of Food-Getting Technology. New York, NY: John Wiley \& Sons, Inc.

Ottoni, E. B., \& Mannu, M. (2001). Semi-free ranging tufted capuchin monkeys (Cebus apella) spontaneously use tools to crack open nuts. International Journal of Primatology, 22(3), 347-358. https://doi.org/10.1023/A:1010747426841

R Core Team, R. (2013). R: a language and environment for statistical computing. Vienna, Austria: R Foundation for Statistical Computing. Retrieved from http://www.r-project.org/

Read, D., \& Andersson, C. (2019). Cultural complexity and complexity evolution. Adaptive Behavior. https://doi.org/10.1177/1059712318822298

Reindl, E., Apperly, I. A., Beck, S. R., \& Tennie, C. (2017). Young children copy cumulative technological design in the absence of action information. Scientific Reports, 7(1778), 1-11. https://doi.org/10.1038/s41598-017-01715-2

Reindl, E., Bandini, E., \& Tennie, C. (2018). The zone of latent solutions and its relation to the classics: Vygotsky and Köhler. In L. D. Di Paolo, F. Di Vincenzo, \& F. De Petrillo (Eds.), Evolution of Primate Social Cognition (pp. 231-248). New York, NY: Springer International Publishing. 
924 Ross, S. R., Milstein, M. S., Calcutt, S. E., \& Lonsdorf, E. V. (2010). Preliminary assessment of 925 methods used to demonstrate nut-cracking behavior to five captive chimpanzees (Pan 926 troglodytes). Folia Primatologica, 81(4), 224-232. https://doi.org/10.1159/000322118

927 Schrauf, C., Call, J., Fuwa, K., \& Hirata, S. (2012). Do chimpanzees use weight to select 928 hammer tools? PLoS ONE, 7(7). https://doi.org/10.1371/journal.pone.0041044

929 Sirianni, G., Mundry, R., \& Boesch, C. (2015). When to choose which tool: multidimensional 930 and conditional selection of nut-cracking hammers in wild chimpanzees. Animal Behaviour, 931 100, 152-165. https://doi.org/10.1016/j.anbehav.2014.11.022

932 Tan, A. W. Y. (2017). From play to proficiency: the ontogeny of stone-tool use in coastal933 foraging long-tailed macaques (Macaca fascicularis) from a comparative perception-action 934 perspective. Journal of Comparative Psychology, 131(2), 89-114. https://doi.org/10.1037/com0000068

Tennie, C., Braun, D. R., Premo, L. S., \& McPherron, S. P. (2016). The island test for 937 cumulative culture in the paleolithic. In Miriam N Haidle, N. J. Conard, \& M. Bolus (Eds.), The Nature of Culture: Based on an Interdisciplinary Symposium 'The Nature of Culture', Tübingen, Germany (pp. 121-133). Dordrecht: Springer Netherlands.

Tennie, C., Caldwell, C. A., \& Dean, L. G. (2018). Culture, Cumulative. In The International Encyclopedia of Anthropology. https://doi.org/10.1002/9781118924396.wbiea1998

943 Tennie, C., Call, J., \& Tomasello, M. (2009). Ratcheting up the ratchet: on the evolution of 944 cumulative culture. Philosophical Transactions of the Royal Society of London. Series B, Biological Sciences, 364(1528), 2405-2415. https://doi.org/10.1098/rstb.2009.0052

946 Tennie, C., Call, J., \& Tomasello, M. (2010). Evidence for emulation in chimpanzees in social 
947 settings using the floating peanut task. PLoS ONE, 5(5).

$948 \quad$ https://doi.org/10.1371/journal.pone.0010544

949 Tennie, C., Call, J., \& Tomasello, M. (2012). Untrained chimpanzees (Pan troglodytes

950 schweinfurthii) fail to imitate novel actions. PLoS ONE, 7(8).

$951 \quad$ https://doi.org/10.1371/journal.pone.0041548

952 Tennie, C., \& Hedwig, D. (2009). How latent solution experiments can help to study differences

953 between human culture and primate traditions. In E. Potocki \& J. Kransinski (Eds.),

954 Primatology: Theories, Methods and Research (pp. 95-112). Hauppauge, NY: Nova

955 Science.

956 Tennie, C., Hedwig, D., Call, J., \& Tomasello, M. (2008). An experimental study of nettle

957 feeding in captive gorillas. American Journal of Primatology, 70(6), 584-593.

958 https://doi.org/10.1002/ajp.20532

959 Thorpe, W. (1963). Learning and Instinct in Animals. (2nd ed.). London: Methuen. Retrieved

960 from https://www.worldcat.org/title/learning-and-instinct-in-animals/oclc/293648

961 Tomasello, M. (1998). Uniquely primate, uniquely human. Developmental Science, 1(1), 1-16.

962 https://doi.org/10.1111/1467-7687.00002

963 Tomasello, M. (1999). The cultural origins of human cognition. Cambridge: Harvard University

964 Press.

965 Tomasello, M., Call, J., Warren, J., Frost, T., Carpenter, M., \& Nagell, K. (1997). The ontoggeny

966 of chimpanzee gestural signals: a comparison across groups and generations. In S. Wilcox

967 (Ed.), Evolution of Communication (pp. 224-259). Amsterdam, The Netherlands: John

968 Benjamins.

969 Tomasello, M., Kruger, A., \& Ratner, H. (1993). Cultural learning. Behavioral and Brain 
971 Vaesen, K., \& Houkes, W. (2017). Complexity and technological evolution: what everybody

972 knows? Biology and Philosophy, 32(6), 1-24. https://doi.org/10.1007/s10539-017-9603-1

973 Van de Waal, E., Borgeaud, C., \& Whiten, A. (2013). Potent social learning and conformity

974 shape a wild primate's foraging decisions. Science, 340(6131), 483-485. https://doi.org/

$975 \quad 10.1126 /$ science. 1232769

976 Visalberghi, E. (1987). Aquisition of nut-cracking behaviour by 2 Capuchin monkeys (Cebus

977 apella). Folia Primatologica, 49, 168-181. https://doi.org/10.1159/000156320

978 Visalberghi, E., Sabbatini, G., Spagnoletti, N., Andrade, F. R. D., Ottoni, E. B., Izar, P., \&

979 Fragaszy, D. M. (2008). Physical properties of palm fruits processed with tools by wild

980 bearded capuchins (Cebus libidinosus). American Journal of Primatology, 70(8), 884-891.

$981 \quad$ https://doi.org/10.1002/ajp.20578

982 Visalberghi, E., Yamakoshi, M. M., Hirata, S., \& Matsuzawa, T. (2002). Responses to novel

983 foods in captive chimpanzees. Zoo Biology, 21(6), 539-548.

984 https://doi.org/10.1002/zoo.10057

985 Whiten, A., Goodall, J., Mcgrew, W. C., Nishida, T., Reynolds, V., Sugiyama, Y., ... Boesch, C.

986 (2001). Charting Cultural Variation in Chimpanzees. Behaviour, 138(11), 1481-1516.

987 https://doi.org/10.1163/156853901317367717

988 Whiten, A., Goodall, J., McGrew, W. C., Nishida, T., Reynolds, V., Sugiyama, Y., ... Boesch, C.

989 (1999). Cultures in chimpanzees. Nature, 399, 15-18. https://doi.org/10.1038/21415

990 Whiten, A., \& Ham, R. (1992). On the nature and evolution of imitation in the animal kingdom:

991 reappraisal of a century of research. Advances in the Study of Behavior, 21, 239-283.

992 https://doi.org/10.1016/S0065-3454(08)60146-1 
993 Whiten, A., Horner, V., Litchfield, C. a, \& Marshall-Pescini, S. (2004). How do apes ape?

994 Learning \& Behavior, 32(1), 36-52. https://doi.org/10.3758/BF03196005 


\section{Table $\mathbf{1}$ (on next page)}

Subject demographic information

Note that subject names are anonymised for the purpose of the study - these codes were kept consistent throughout the study. Subject $\mathrm{C13}$ is displayed in italics as she was only included in the study after the baseline condition. 
1

\begin{tabular}{lllcll}
\hline ID & Sub-Species & DoB & Age (first testing day) & Sex & Rearing \\
\hline C1 & Hybrid & $30 / 04 / 1976$ & 41 & Female & Hand \\
C2 & Hybrid & $09 / 06 / 1982$ & 35 & Male & Hand \\
C3 & Hybrid & $25 / 10 / 1986$ & 31 & Male & Hand \\
C4 & Hybrid & $18 / 08 / 1990$ & 27 & Female & Hand \\
C5 & Hybrid & $28 / 12 / 1990$ & 26 & Male & Hand \\
C6 & Hybrid & $10 / 08 / 2007$ & 10 & Female & Parent \\
C7 & Hybrid & $25 / 05 / 1995$ & 22 & Female & Hand \\
C8 & Schweinfurthii & $17 / 06 / 1977$ & 40 & Female & Undetermined \\
C9 & Verus & $20 / 02 / 1988$ & 29 & Female & Hand \\
C10 & Verus & $01 / 01 / 1965$ & 52 & Female & Undetermined \\
C11 & Verus & $14 / 12 / 1971$ & 45 & Female & Undetermined \\
C12 & Verus & $05 / 12 / 2003$ & 13 & Male & Parent \\
C13 & Hybrid & $27 / 12 / 1982$ & 34 & Female & Parent \\
\hline
\end{tabular}

2 


\section{Table 2 (on next page)}

Coding ethogram used during the live coding procedure.

This was added to throughout live coding as behaviours of interest were observed. This ethogram was provided to the second coder for reliability coding. 
1

\begin{tabular}{ll}
\hline Behaviour & Description \\
\hline Place nut & $\begin{array}{l}\text { The subject places one/several nuts on the surface of the anvil. This is } \\
\text { also coded if the subject drops the nut onto the anvil. The nut may roll } \\
\text { off the anvil after being "placed" this is acceptable as it is likely due to } \\
\text { the nut's shape and the angle of the anvil's surface. }\end{array}$ \\
The subject picks up the hammer - with the nut on the anvil, by holding \\
the wood itself or the securing attachment. \\
Raise hammer $\quad \begin{array}{l}\text { The subject lifts the hammer above the nut - this may be at/below/above } \\
\text { head height for the subject. }\end{array}$ \\
The subject brings the hammer down onto the nut, which must be \\
resting on the anvil. The hammer can be dropped or held in the hand the \\
entire time. This behaviour can be repeated until the nut is cracked. The \\
behaviour is coded each time the behaviour occurs - i.e., each time the \\
nut is struck. \\
The subject takes the kernel of the, now broken nut and eats it. Note, \\
this must have followed cracking of the nut by the subject. \\
The subject uses their foot to stamp on the nut, which has been placed \\
on the anvil.
\end{tabular}

* Note that throw did not require any behaviours preceding it 


\section{Figure 1}

Decision tree depiction of the result dependent conditions.

If, at any stage, evidence of the behaviour was encountered then testing would cease, and the resultant learning mechanism will be attributed to the emergence of the behaviour. Each condition is continued for five trials before moving onto the next condition.

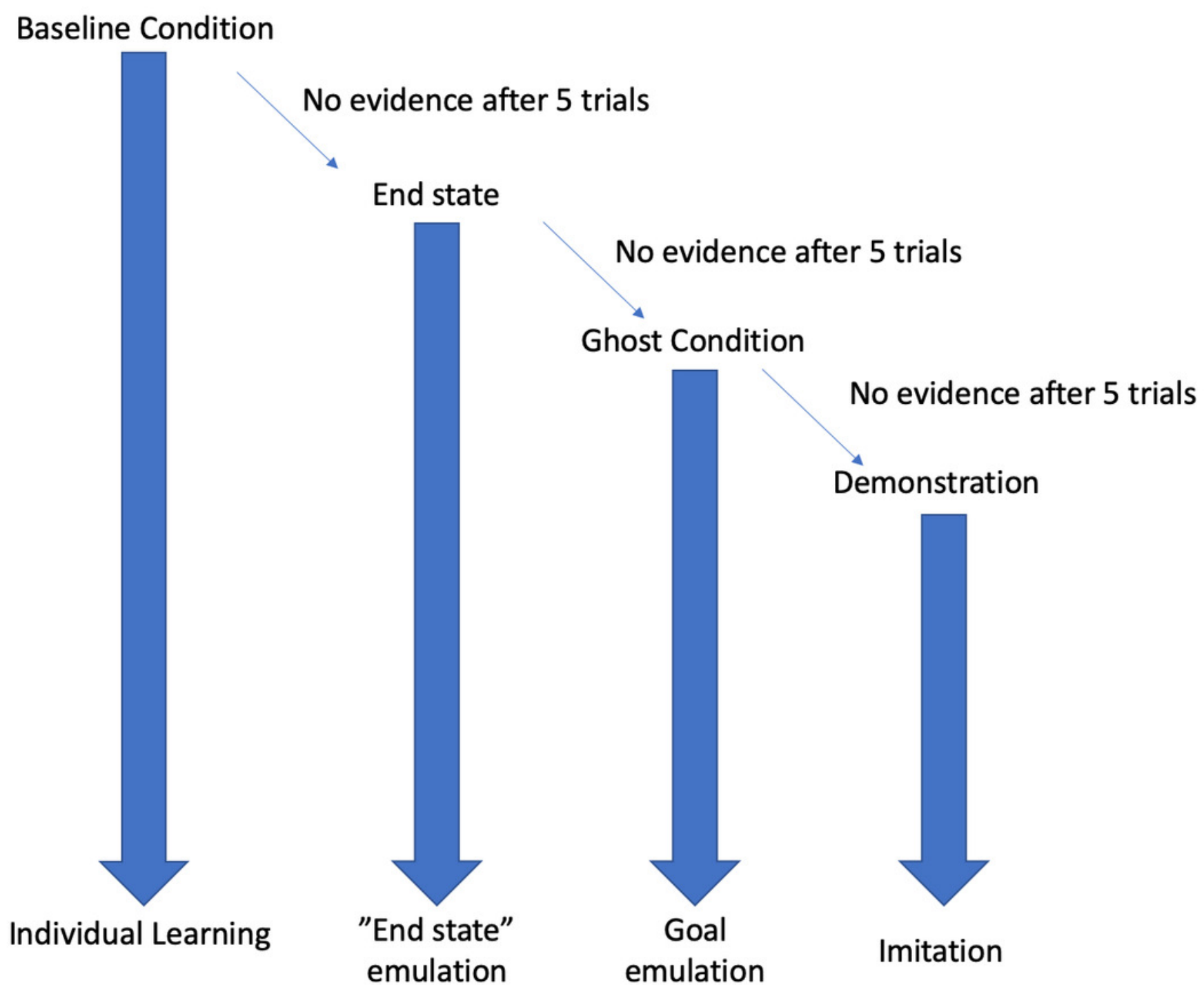


Figure 2

Securing attachment of the hammer.

Note how there are several 'clips' to act as a failsafe.

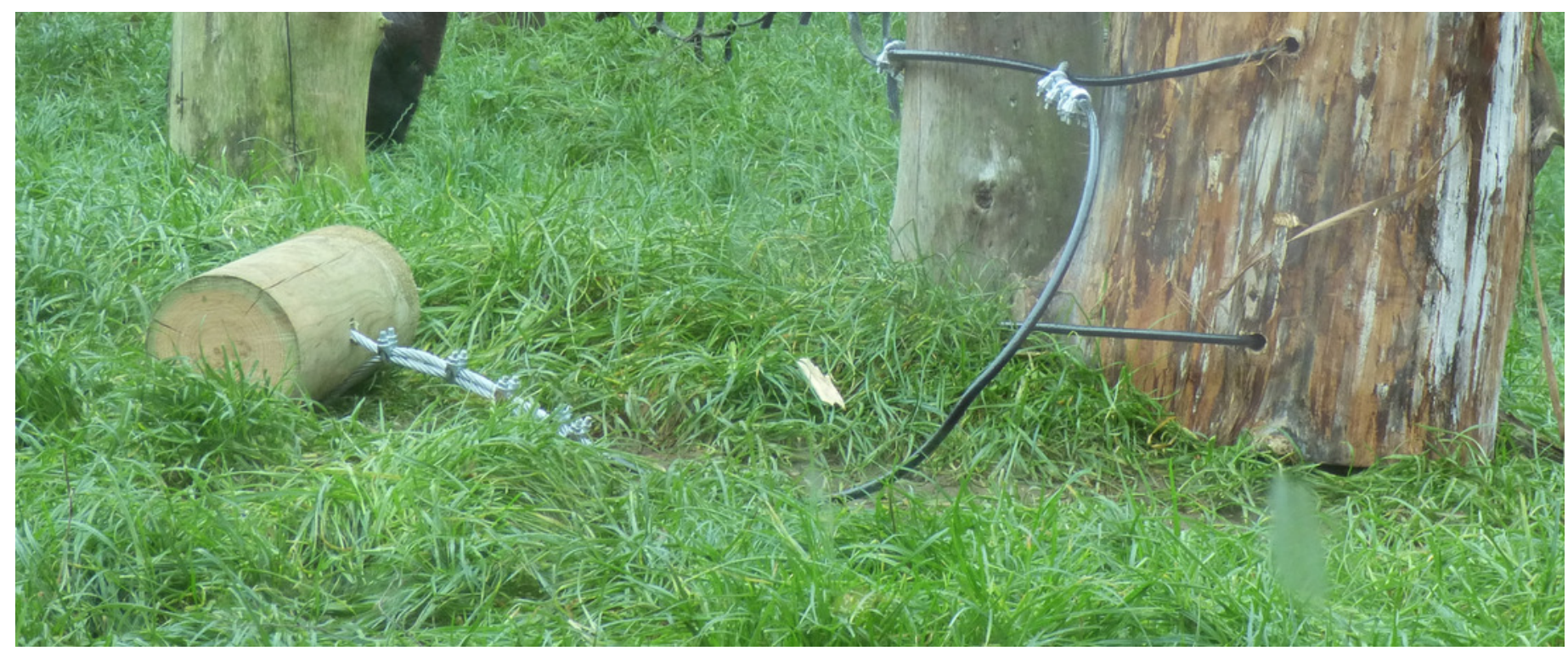




\section{Figure 3}

Hammer and anvil set up within the subjects ' enclosure.

Note, the two securing attachments are passed through separate holes within the anvil and the hammer is less than $1 \mathrm{~m}$ from the anvil (this was ensured by the length of the securing attachment of the hammer to the anvil).

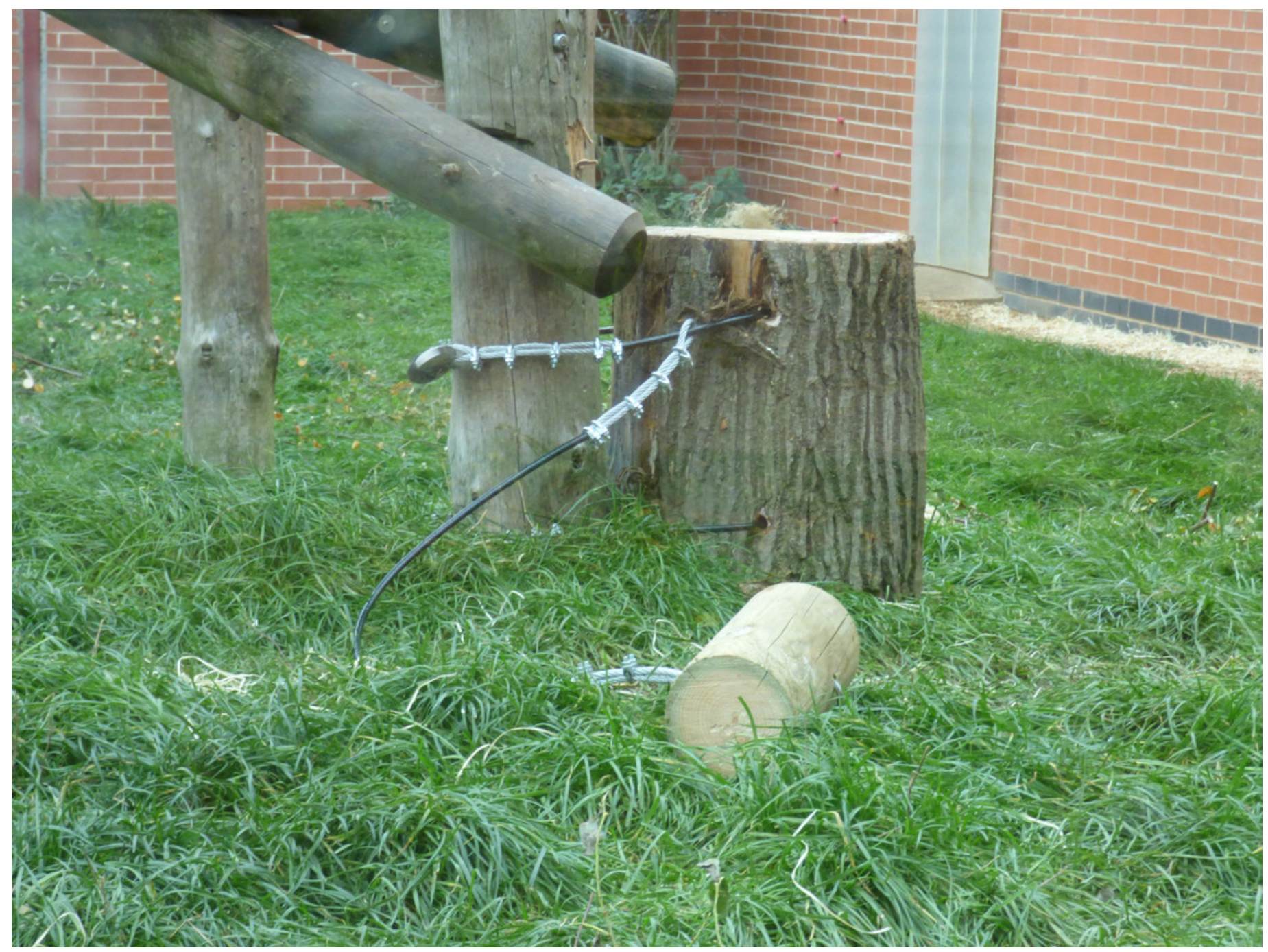


Figure 4

Experimental set up for baseline condition in "conversion"

Note, the same set up was used for the first two trials of the "end-state" condition, prior to the enclosure move (see below).

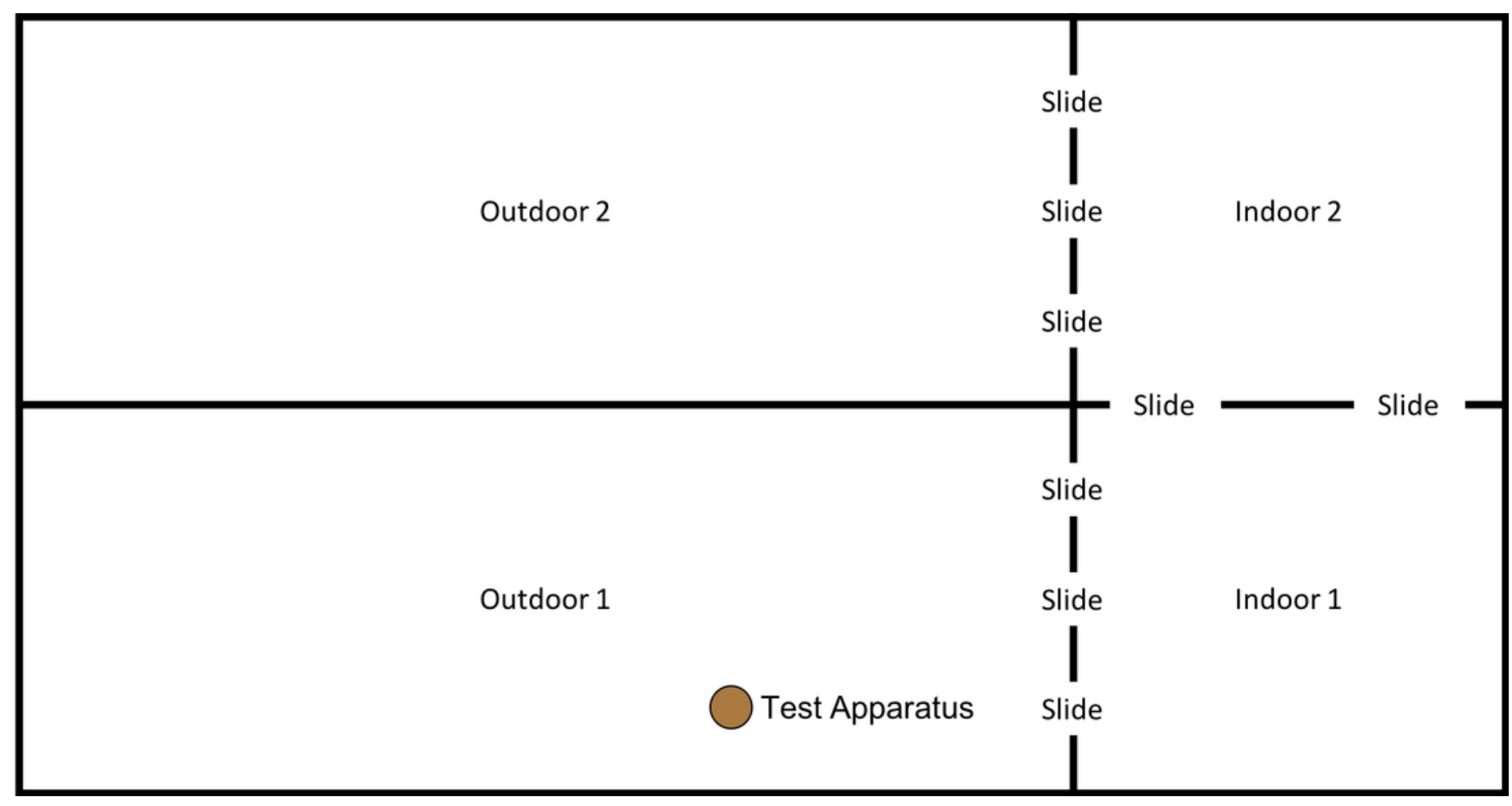


Figure 5

Experimental set up for "end-state" condition in "Eden".

Note, subjects had access to the entirety of this enclosure throughout these trials, however, the outdoor section of the enclosure was still under construction.

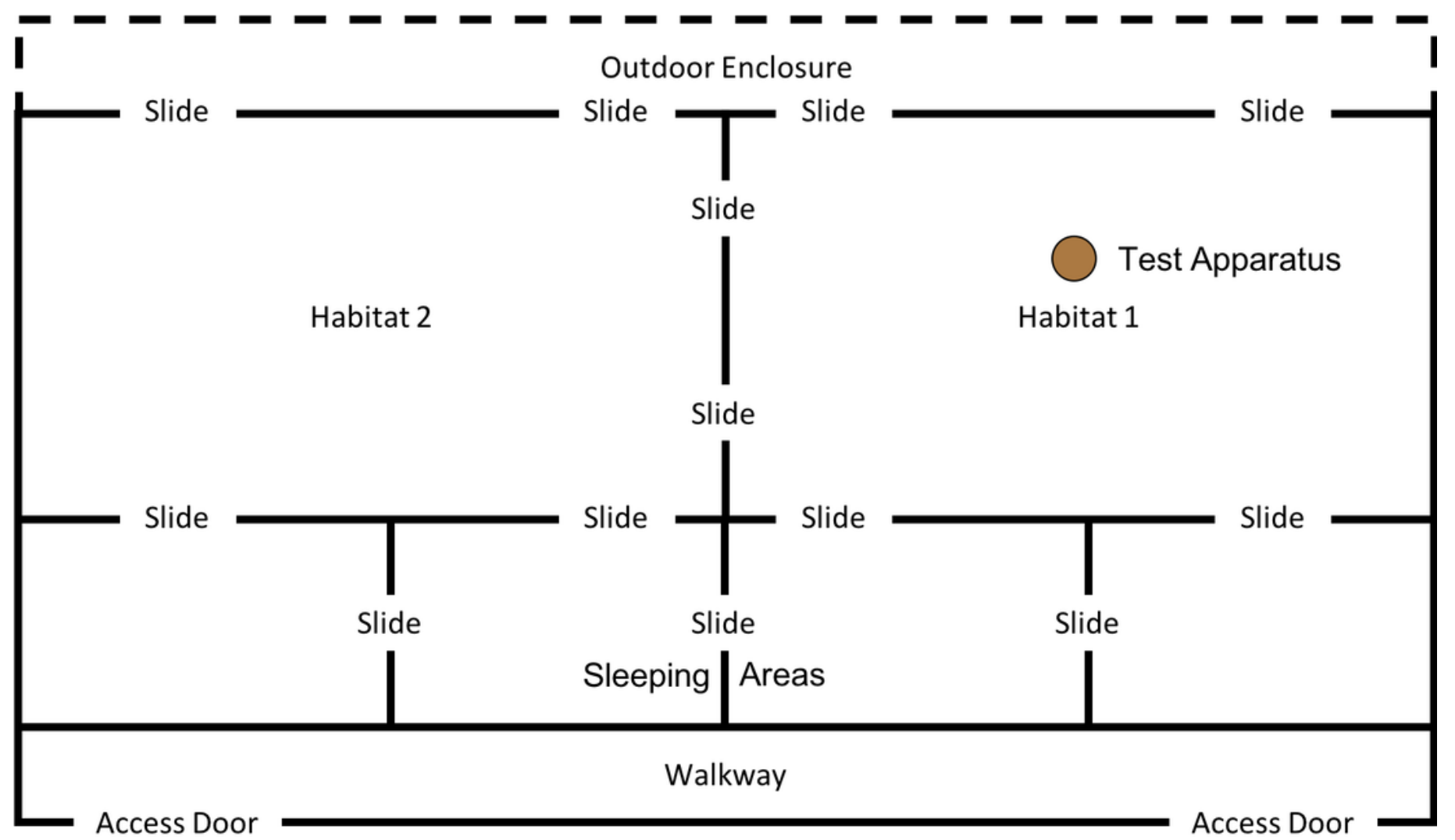




\section{Figure 6}

Macadamia nut placement and state for end-state condition.

(A) Macadamia nuts, sawn in half (with kernels left whole) for the end-state emulation condition. (B) Nuts placed atop the anvil as described in text.

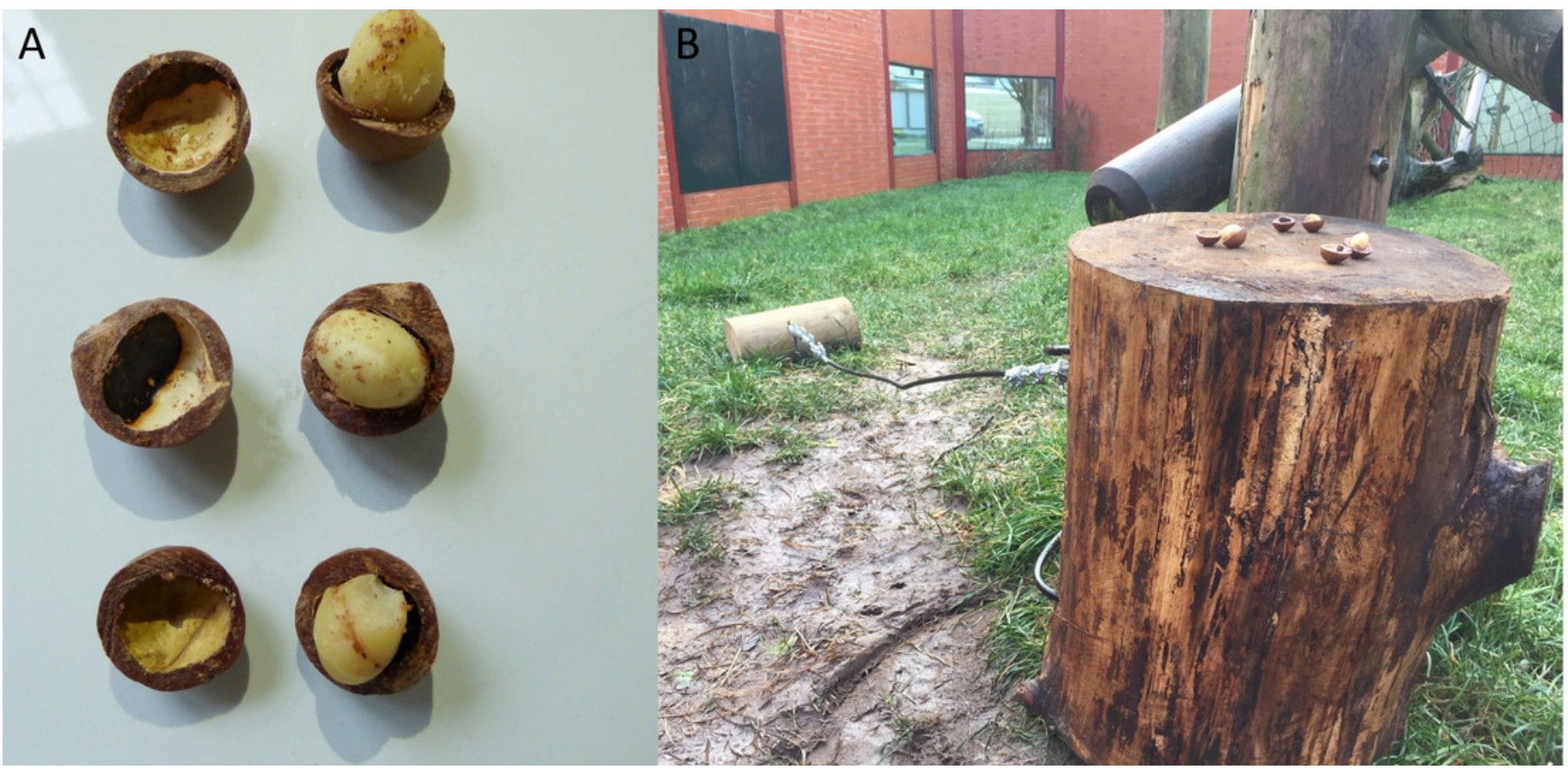




\section{Figure 7}

Adult male chimpanzee (C5) cracking a macadamia nut with his teeth, then eating the kernel.

(A) $\mathrm{C} 5$ biting the nut in an attempt to break it; (B) C5 consuming the kernel from the now broken shell

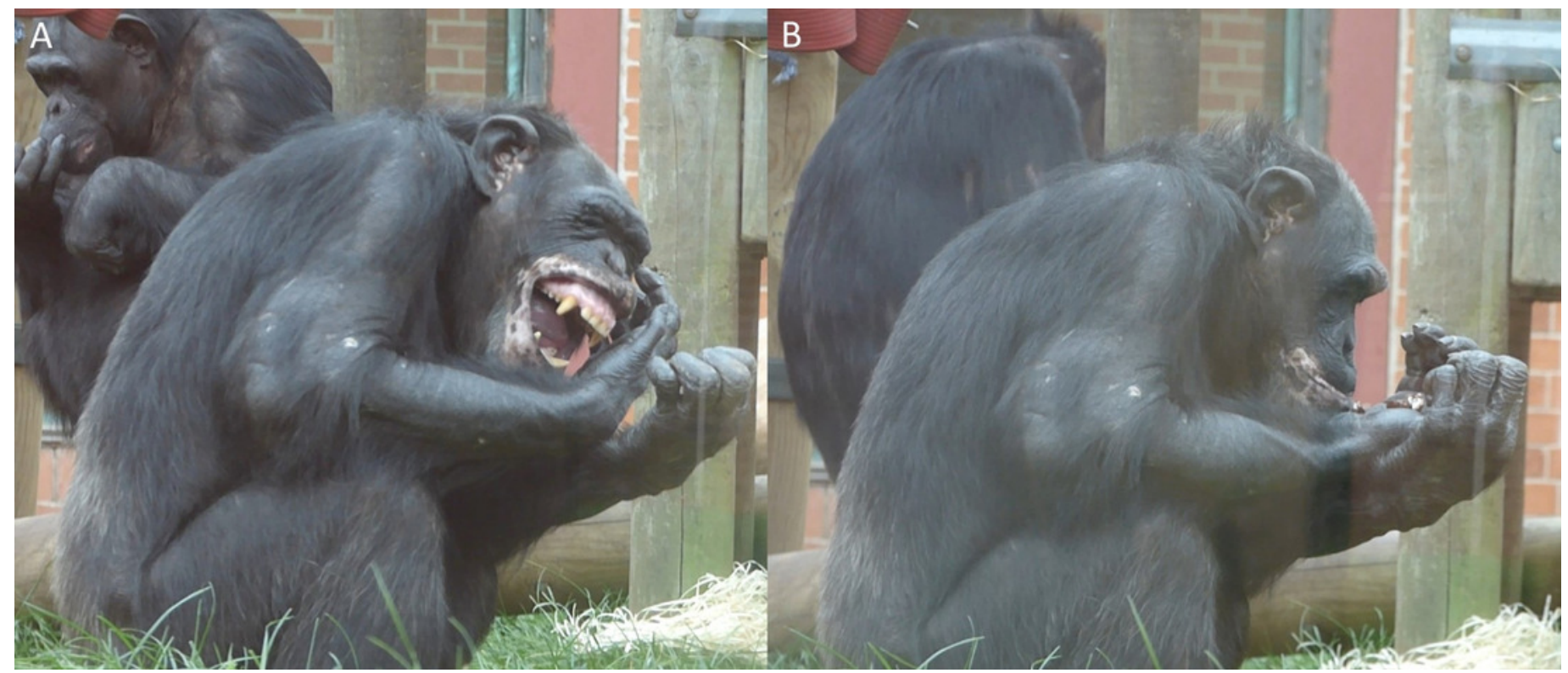


Figure 8

Experimental set up for "Ghost" condition in "Eden".

Note, subjects had access to the entirety of this enclosure throughout these trials including the outdoor enclosure.

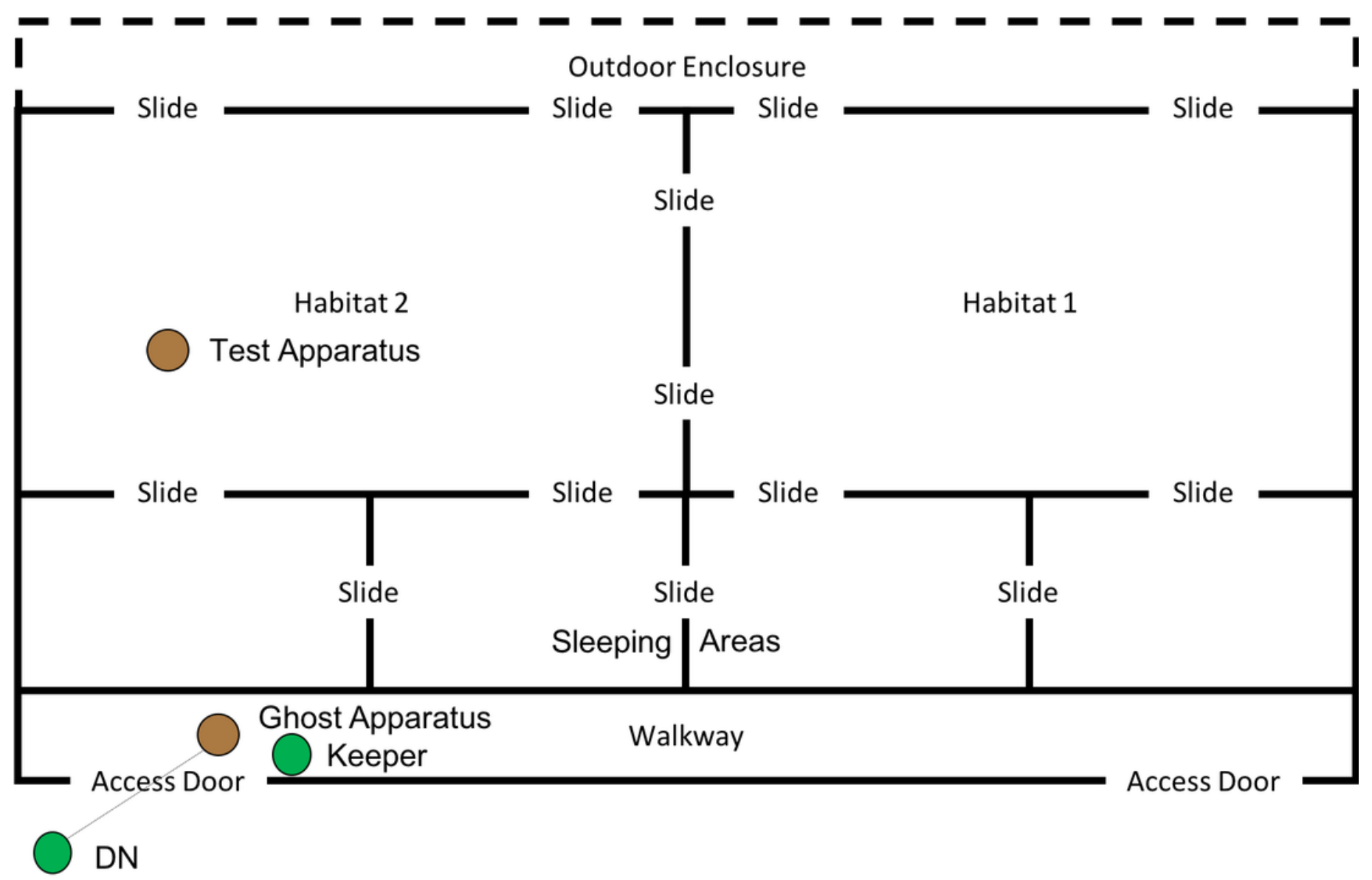




\section{Figure 9}

Experimental set up of "Ghost Condition" apparatus.

Note, the hammer is suspended by fishing line, and a single nut is in the centre of the anvil.

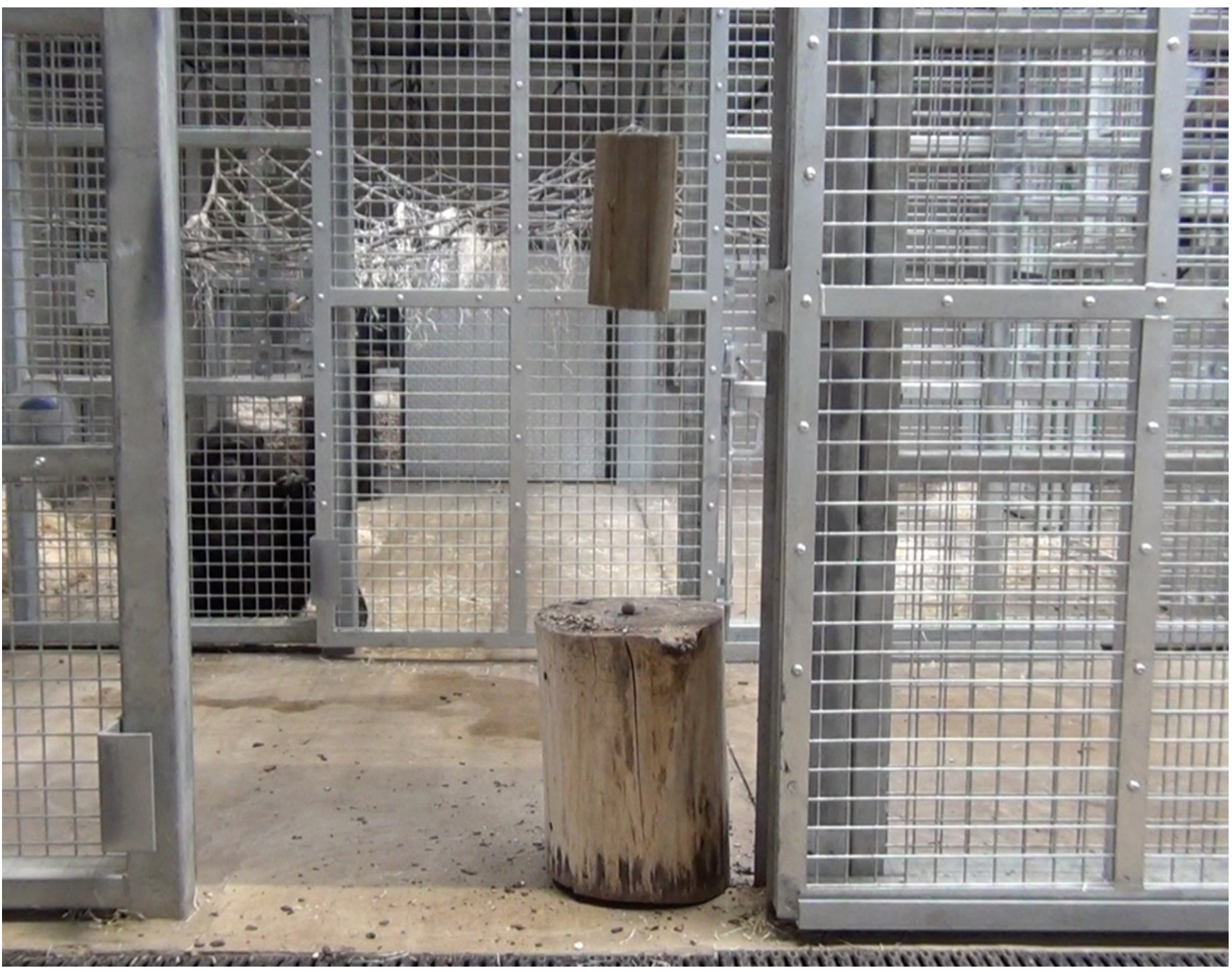




\section{Figure 10}

DN performing full nut-cracking action demonstrations

(A) subjects' attention was gained by calling their given name; (B) hammer was to eye-level and $(C)$ brought down on the nut as many times as required until it cracked; $(D)$ the cracked nut (both shell and kernel) are provided to the target subject by a keeper. Subject in this demonstration was the female (C9) to the left of DN - holding onto the mesh in panels A-C; keeper rolled the nut to C9 in panel D (hand feeding, even by keepers, is not permitted at the testing institution).

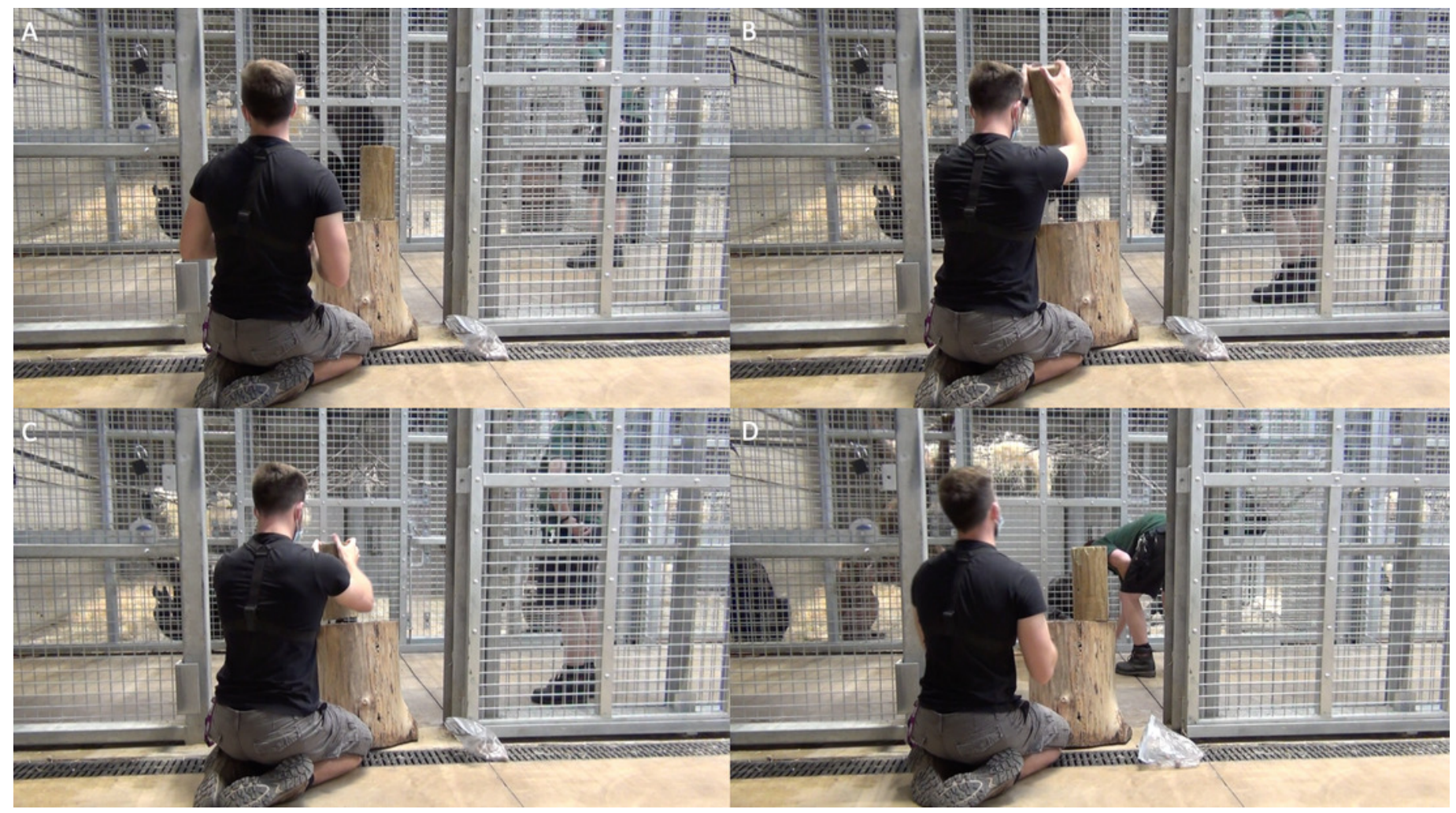

